\title{
NEW TYPES OF COMPLETENESS IN METRIC SPACES
}

\author{
M. Isabel Garrido and Ana S. Meroño
}

\author{
Universidad Complutense de Madrid, Instituto de Matemática Interdisciplinar (IMI) \\ Departamento de Geometría y Topología, 28040 Madrid, Spain; maigarri@mat.ucm.es \\ Universidad Complutense de Madrid, Departamento de Geometría y Topología \\ 28040 Madrid, Spain; anamerogno@gmail.com
}

\begin{abstract}
This paper is devoted to introduce and study two new properties of completeness in the setting of metric spaces. We will call them Bourbaki-completeness and cofinal Bourbakicompleteness. These notions came from new classes of generalized Cauchy sequences appearing when we try to characterize the so-called Bourbaki-boundedness in a similar way that Cauchy sequences characterize the totally boundedness. We also study the topological problem of metrizability by means of a Bourbaki-complete or a cofinally Bourbaki-complete metric. At this respect, we obtain results in the line to the classical Čech theorem about the complete metrizability of a metric space $X$ in terms of its Stone-Čech compactification $\beta X$. Finally we give some relationships between these kinds of completeness and some properties related to paracompactness and uniform paracompactness in the framework of metrizable spaces.
\end{abstract}

\section{Introduction}

Some classes of metric spaces satisfying properties stronger than completeness but weaker than compactness have been recently studied by many authors. A good reference for this topic is the nice paper by Beer [5], entitled "Between compactness and completeness". Examples of these properties are the bounded compactness, the uniform local compactness, the cofinal completeness, the strong cofinal completeness, recently introduced by Beer in [6], as well as the so-called UC-ness for metric spaces. The study of all these spaces have shown to be not only interesting by themselves but also in connection with some problems in Convex Analysis, in Optimization Theory and in the setting of Convergence Structures on Hyperspaces (see for instance [7] and references therein).

The general aim of this paper is to introduce a new couple of these intermediate properties in the context of metric spaces. First of all, note that a way to achieve a property stronger than completeness for a metric space consists of asking for the clustering of all the sequences belonging to some class bigger than the class of Cauchy sequences. Thus, in Section 2, we define the class of Bourbaki-Cauchy sequences and the class of cofinally Bourbaki-Cauchy sequences. These sequences appear when we consider in a metric space the so-called Bourbaki-bounded sets. This notion of boundedness was introduced by Atsuji in [2] in order to exhibit metric spaces where every real-valued uniformly continuous function is bounded but they are not necessarily totally bounded. The name of Bourbaki-bounded cames from the book of Bourbaki [8], where these subsets in uniform spaces is considered. We prove here

doi:10.5186/aasfm.2014.3934

2010 Mathematics Subject Classification: Primary 54E35, 54E50, 54E15, 54D35, 54D20.

Key words: Metric spaces, Bourbaki-completeness, cofinal Bourbaki-completeness, Bourbakibounded sets, totally-bounded sets, Stone-Čech compactification, uniform paracompactness.

The first author has been supported in part by MINECO Project-MTM2012-34341 (Spain). 
that the Bourbaki-bounded subsets of a metric space can be characterized in terms of sequences in the same way that Cauchy sequences characterize total boundedness. Thus, a new type of sequences appears that we call Bourbaki-Cauchy sequences.

Next, we generate another class of sequences which are cofinal with respect to the previous ones, in the sense that the residuality of the indexes is replaced by the cofinality. Then, we obtain what we call cofinally Bourbaki-Cauchy sequences. Recall that the corresponding cofinal notion associated to the usual Cauchy sequences was defined by Beer in [5], in the following way. A sequence $\left(x_{n}\right)_{n \in \mathbf{N}}$ in a metric space $(X, d)$ is called cofinally Cauchy if for every $\varepsilon>0$ there exists an infinite subset $\mathbf{N}_{\varepsilon}$ of $\mathbf{N}$ such that for each $i, j \in \mathbf{N}_{\varepsilon}$ we have $d\left(x_{i}, x_{j}\right)<\varepsilon$.

Now, if we ask for the clustering of Bourbaki-Cauchy sequences and cofinally Bourbaki-Cauchy sequences, we have respectively what we will call Bourbaki-completeness and cofinal Bourbaki-completeness of metric spaces. Thus, in Section 3, we present a detailed study of both of these properties showing, in particular, that they are stronger than the usual completeness but weaker than compactness, and also that they are mutually different. We prove also that they are in fact weaker than the property of uniform local compactness. And this will be interesting along the paper, since we will see that the set of points in a metric space having no compact neighborhood will play an important role in this context.

On the other hand, a property which is given in terms of sequences in metric spaces, has to be reformulated in terms of nets and entourages when we want to extend it to the more general setting of uniform spaces. For example, that happens when we define a uniform space to be complete whenever every Cauchy net clusters (converges). It is not difficult to see, and interesting to note here, that a metric space is complete (by sequences) if, and only if, it is complete as a uniform space (i.e., by nets) (see, for instance [34]). Thus, in Section 4, we introduce the corresponding notions of Bourbaki-Cauchy net and Bourbaki-Cauchy completeness for uniform spaces. We will see that also for Bourbaki-completeness is equivalent to use sequences or nets for metrizable uniformities. The proof of this result (Theorem 19) is not so elemental as for the usual completeness. We present here a proof that Hohti and Junnila (University of Helsinki) have personally communicated to us after we have had some discussions about this topic. Finally, note that the corresponding result for cofinal Bourbaki-completeness is also true as we can see in the last section (Theorem 28).

In sections 5 and 6 , we consider the natural problem of metrization in this setting. That is, we wonder when a metrizable space admits a Bourbaki-complete or a cofinally Bourbaki-complete equivalent metric. At this respect, we obtain results (Theorems 23 and 34) in the line of the classical theorem by Cech saying that a a metric space is completely metrizable if, and only if, it is a $G_{\delta^{-}}$set in its Stone-Čech compactification ([12]).

The rest of the paper is devoted to compare our completeness notions with some properties of paracompactness and uniform paracompactness in the framework of metrizable spaces. For this purpose the notion of cofinal completeness will be of special relevance. Recall that a metric space is said to be cofinally complete whenever every cofinally Cauchy sequence clusters. Cofinal completeness was introduced implicitly by Corson in [13] in the frame of uniform spaces in order to characterize paracompactness and Lindelöfness as filter properties. In fact, he proved that a 
topological space is paracompact if there exists some compatible uniformity for which some kind of filters (weakly Cauchy) cluster. Afterwards, Howes [23] defined cofinal completeness in uniform spaces by means of cofinally Cauchy nets. In parallel, Rice [30] defines and studies some (equivalent) uniform extensions of paracompactness for uniform spaces. According to this paper we say that a metric space $(X, d)$ is uniformly paracompact if for every open cover $\mathcal{U}$ there exists an open refinement and some $\delta>0$ such that for every $x \in X$ the open ball $B_{\delta}(x)$ of center $x \in X$ and radius $\delta$, meets only finitely many members of the refinement. But it was Smith [32], the reviewer of Rice's paper, who noticed that the property of uniform paracompactness is in fact equivalent to cofinal completeness for uniform spaces. On the other hand, cofinal completeness as well as uniform paracompactness have been considered in the frame of metric spaces by authors like Rice [30], Hohti [22], and Romaguera [31]. More recently, Beer in [5] has studied cofinal completeness of metric spaces in terms of sequences. In particular, he pointed out that sequential cofinal completeness and net cofinal completeness are again equivalent for metric spaces, since both properties are indeed equivalent to the condition given by Hohti [22] to uniform paracompactness in this context.

Then, motivated by the mentioned equivalence between cofinal completeness and uniform paracompactness, we study in the two last sections how our completeness properties are related with different paracompactness notions for metric spaces. First of all, we will see in Section 5 interesting relationships between Bourbaki-complete metrizability with some other topological properties like strong paracompactness, strong metrizability and also with the notion of $\delta$-completeness given García-Máynez in [16]. Finally, in Section 6, we will prove as a main result (Theorem 32) that cofinal Bourbaki-completeness and the uniform version of strong paracompactness introduced by Hohti in [22], are equivalent for metric spaces.

\section{Bourbaki-Cauchy and cofinally Bourbaki-Cauchy sequences}

We start with some notation. For a metric space $(X, d)$, denote by $B_{\varepsilon}(x)$ the open ball of center $x \in X$ and radius $\varepsilon>0$. And, for any subset $A$ of $X$ and $\varepsilon>0$ we will write the $\varepsilon$-enlargement of $A$ by,

$$
A^{\varepsilon}=\bigcup\left\{B_{\varepsilon}(x): x \in A\right\}=\{y: d(y, A)<\varepsilon\} .
$$

Along the paper we will use the subsequent $n^{\text {th }} \varepsilon$-enlargement of the open ball $B_{\varepsilon}(x)$, defined as follows: $B_{\varepsilon}^{1}(x)=B_{\varepsilon}(x)$ and for every $n \geq 2$,

$$
B_{\varepsilon}^{n}(x):=\left(B_{\varepsilon}^{n-1}(x)\right)^{\varepsilon} .
$$

Finally, we define the $\varepsilon$-chainable component on $x \in X$ by,

$$
B_{\varepsilon}^{\infty}(x):=\bigcup_{n \in \mathbf{N}} B_{\varepsilon}^{n}(x) .
$$

Recall that in a metric space a subset is said to be (metric) bounded if it has finite diameter, that is, when it is contained in some open ball. This notion of boundedness is quite natural but it has some inconvenience. Namely, to be metric bounded is not a uniform invariant, that is, it is not preserved under uniform homeomorphisms. However, in the setting of normed spaces metric boundedness is a uniform property and in fact the bounded subsets can be characterized by means of uniformly continuous functions. Indeed, it is easy to see that a subset $B$ of a normed space $X$ 
is bounded by the norm if, and only if, for every real-valued uniformly continuous function $f$ on $X$, we have that $f(B)$ is bounded in $\mathbf{R}$ endowed with its usual metric. As we have said in the introduction, it was Atsuji who introduced in [2] the notion of Bourbaki-bounded metric space (under the name of finitely-chainable metric space) and he showed that they are just the metric spaces having bounded image under every real-valued uniformly continuous function defined on it. These spaces were also considered in the frame of uniform spaces by Hejcman in [21] who called them simply as bounded.

Definition 1. A subset $B$ of a metric space $(X, d)$ is said to be a Bourbakibounded subset of $X$ if for every $\varepsilon>0$ there exist $m \in \mathbf{N}$ and a finite collection of points $p_{1}, \ldots, p_{k} \in X$ such that,

$$
B \subset \bigcup_{i=1}^{k} B_{\varepsilon}^{m}\left(p_{i}\right) .
$$

Note that the family B of all Bourbaki-bounded subsets of $X$ forms a bornology in $X$, that is, $\mathbf{B}$ satisfies the following conditions: (i) for every $x \in X$, the set $\{x\} \in \mathbf{B}$; (ii) if $B \in \mathbf{B}$ and $A \subset B$, then $A \in \mathbf{B}$; and (iii) if $A, B \in \mathbf{B}$ then $A \cup B \in \mathbf{B}$.

On the other hand, if, in the above Definition 1, we have $m=1$ (or even $m \leq$ $m_{0}$, for some $m_{0} \in \mathbf{N}$ ), for every $\varepsilon>0$, then we recover the classical notion of totally bounded subset. Thus, every totally bounded subset is in particular Bourbakibounded. And, it is easy to see that any Bourbaki-bounded subset is in particular bounded in the usual sense. For further information about the relationships among these three different properties of boundedness we refer to [19] and [20], where we study all of them into the general setting of the so-called bornological universes.

It is important to note here that to be a Bourbaki-bounded subset is not an intrinsic property, i.e., it depends on the ambient space where we are. In fact, we will see that a subset can be Bourbaki-bounded in $X$ but not in itself (Example 6 ). When $X$ is a Bourbaki-bounded subset of itself, we say that $X$ is a Bourbaki-bounded metric space.

The next result collects (without proofs) some nice characterizations of Bourbakiboundedness, given by different authors. Recall that a cover $\mathcal{A}$ of a set $X$ is star-finite if every $A \in \mathcal{A}$ meets only finitely many $A^{\prime} \in \mathcal{A}$.

Theorem 2. Let $(X, d)$ be a metric space, the following statements are equivalent:

(1) $X$ is a Bourbaki-bounded metric space.

(2) (Atsuji, [2]) For every real uniformly continuous function $f$ on $X, f(X)$ is bounded in $\mathbf{R}$.

(3) (Hejcman, [21]) $X$ is d'-bounded, for every metric d' uniformly equivalent to $d$.

(4) (Njåstad, [28]) Every star-finite uniform cover of $X$ is finite.

Now we are going to give a characterization by sequences of the Bourbakibounded subsets of a metric space. Firstly we need to introduce the announced notions of Bourbaki-Cauchy and cofinally Bourbaki-Cauchy sequences.

Definition 3. Let $(X, d)$ be a metric space. A sequence $\left(x_{n}\right)_{n \in \mathbf{N}}$ is said to be Bourbaki-Cauchy in $X$ if for every $\varepsilon>0$ there exist $m \in \mathbf{N}$ and $n_{0} \in \mathbf{N}$ such that for some $p \in X$ we have that $x_{n} \in B_{\varepsilon}^{m}(p)$, for every $n \geq n_{0}$. On the other hand, $\left(x_{n}\right)_{n \in \mathbf{N}}$ 
is said to be cofinally Bourbaki-Cauchy in $X$ if for every $\varepsilon>0$ there exist $m \in \mathbf{N}$ and an infinite subset $\mathbf{N}_{\varepsilon} \subset \mathbf{N}$ such that for some $p \in X$ we have that $x_{n} \in B_{\varepsilon}^{m}(p)$, for every $n \in \mathbf{N}_{\varepsilon}$.

Theorem 4. For a metric space $(X, d)$ and $B \subset X$, the following statements are equivalent:

(1) $B$ is a Bourbaki-bounded subset in $X$.

(2) Every countable subset of $B$ is a Bourbaki-bounded subset in $X$.

(3) Every sequence in $B$ has a Bourbaki-Cauchy subsequence in $X$.

(4) Every sequence in $B$ is cofinally Bourbaki-Cauchy in $X$.

Proof. (1) $\Rightarrow(2)$ By definition it is clear that every subset of a Bourbaki-bounded subset of $X$ is also a Bourbaki-bounded subset of $X$.

$(2) \Rightarrow(3)$ Let $\left(x_{n}\right)_{n \in \mathbf{N}}$ be a sequence in $B$. By the hypothesis, the set $\left\{x_{n}: n \in\right.$ $\mathbf{N}$ \} is Bourbaki-bounded in $X$, and then for $\varepsilon=1$ there exist $m_{1} \in \mathbf{N}$ and some points $p_{1}^{1}, \ldots, p_{j_{1}}^{1} \in X$ such that,

$$
\left\{x_{n}: n \in \mathbf{N}\right\} \subset \bigcup\left\{B_{1}^{m_{1}}\left(p_{i}^{1}\right): i=1, \ldots, j_{1}\right\} .
$$

Since the family $\left\{B_{1}^{m_{1}}\left(p_{i}^{1}\right): i=1, \ldots, j_{1}\right\}$ is finite, then some $B_{1}^{m_{1}}\left(p_{i_{1}}^{1}\right)$ contains infinite terms of the sequence. Therefore, there exists a subsequence $\left(x_{n}^{1}\right)_{n \in \mathbf{N}}$ of $\left(x_{n}\right)_{n \in \mathbf{N}}$ inside to $B_{1}^{m_{1}}\left(p_{i_{1}}^{1}\right)$.

Repeating this process we have that, for every $k \geq 2$ and $\varepsilon=1 / k$, there exist some $m_{k} \in \mathbf{N}$ and points $p_{1}^{k}, \ldots, p_{j_{k}}^{k} \in X$, such that $\left\{B_{1 / k}^{m_{k}}\left(p_{i}^{k}\right): i=1, \ldots, j_{k}\right\}$ is a finite cover of $\left\{x_{n}^{k-1}: n \in \mathbf{N}\right\}$. Then there exist some $B_{1 / k}^{m_{k}}\left(p_{i_{k}}^{k}\right)$ containing some subsequence $\left(x_{n}^{k}\right)_{n \in \mathbf{N}}$ of $\left(x_{n}^{k-1}\right)_{n \in \mathbf{N}}$.

Finally, choosing the standard diagonal subsequence $\left(x_{n}^{n}\right)_{n \in \mathbf{N}}$ we can check, in an easy way, that it is the required Bourbaki-Cauchy subsequence of $\left(x_{n}\right)_{n \in \mathbf{N}}$.

(3) $\Rightarrow(4)$ Trivial.

$(4) \Rightarrow(1)$ Suppose that $B$ is not a Bourbaki-bounded subset of $X$. Then, there exists $\varepsilon_{0}>0$ such that, for every $m \in \mathbf{N}$, the family $\left\{B_{\varepsilon_{0}}^{m}(x): x \in X\right\}$ does not contain any finite subcover of $B$. Fix $x_{0} \in X$ and for every $m \in \mathbf{N}$ choose $x_{m} \in B$ such that $x_{m} \notin B_{\varepsilon_{0}}^{m}\left(x_{i}\right), i=0, . ., m-1$. Then, the sequence $\left(x_{m}\right)_{m \in \mathbf{N}}$ constructed in this way is not a cofinally Bourbaki-Cauchy sequence in $X$. Otherwise, for this $\varepsilon_{0}$ there must exist $m_{0} \in \mathbf{N}$ and an infinite subset $\mathbf{N}_{\varepsilon_{0}} \subset \mathbf{N}$ such that for some $p_{0} \in X$ we have that $x_{n} \in B_{\varepsilon_{0}}^{m_{0}}\left(p_{0}\right)$, for every $n \in \mathbf{N}_{\varepsilon_{0}}$. Then taking $n_{0} \in \mathbf{N}_{\varepsilon_{0}}$, we have that there are infinitely many terms of the sequence $\left(x_{m}\right)_{m \in \mathbf{N}}$ in $B_{\varepsilon_{0}}^{2 \cdot m_{0}}\left(x_{n_{0}}\right)$, which is a contradiction.

Recall the classical result saying that, in a metric space $(X, d)$, a subset $B \subset X$ is totally bounded if, and only if, every sequence in $B$ has a Cauchy subsequence (see for instance [34]). Hence the equivalence between (1) and (3) in last result is the analogous characterization for Bourbaki-boundedness. Moreover, in [5] Beer also characterizes the totally bounded metric spaces as those in which every sequence is cofinally Cauchy. Therefore, the above equivalence between (1) and (4) can be seen as the corresponding result for Bourbaki-bounded metric spaces.

On the other hand, since every subsequence of a Bourbaki-Cauchy sequence in $X$ is also Bourbaki-Cauchy in $X$ then, we can derive from the above Theorem 4 that every Bourbaki-Cauchy sequence is in particular a Bourbaki-bounded subset. 
Note that a similar result does not hold for cofinally Bourbaki-Cauchy sequences (Example 5).

Finally, note that clearly every Cauchy sequence is Bourbaki-Cauchy, every cofinally Cauchy sequence is cofinally Bourbaki-Cauchy and every Bourbaki-Cauchy sequence is cofinally Bourbaki-Cauchy. But the reverse implications are not true, as we can see in the following examples.

Example 5. In the real line $\mathbf{R}$, with the usual metric, consider the sequence

$$
q_{1}, 1, q_{2}, 2, q_{3}, 3, \ldots, q_{n}, n, \ldots
$$

where $\left\{q_{1}, q_{2}, \ldots, q_{n}, \ldots\right\}$ is some enumeration of the set $\mathbf{Q} \cap(0,1)$. Then this sequence is not a bounded set, and then it is not a Bourbaki-bounded subset nor a Bourbaki-Cauchy sequence in $\mathbf{R}$. On the other hand, it is easy to check that it is a cofinally Cauchy (not Cauchy) sequence and hence it is also cofinally Bourbaki-Cauchy sequence in $\mathbf{R}$.

The next example shows that also to be a Bourbaki-Cauchy sequence or a cofinally Bourbaki-Cauchy sequence depends on the whole space. This is the reason for which we must always specify the ambient space whenever we deal with any kind of Bourbaki notion.

Example 6. Let $\left(\ell_{2},\|\cdot\|\right)$ be the classical Hilbert space of all the real square summable sequences, and let $B=\left\{e_{n}: n \in \mathbf{N}\right\}$ its standard basis. It is easy to see that $B$ (and, in general, every bounded subset in a normed space) is Bourbakibounded in the whole space. Indeed, for every $\varepsilon>0$, let $m>1 / \varepsilon$, then $B \subset B_{\varepsilon}^{m}(0)$. Nevertheless, $B$ is not a Bourbaki-bounded subset of itself, because it is an infinite uniformly discrete metric space. In fact, in the metric space $\left(B,\|\cdot\|_{B}\right)$ and for $\varepsilon=1$ we have that $B_{\varepsilon}^{m}\left(e_{n}\right)=\left\{e_{n}\right\}$, for every $m, n \in \mathbf{N}$. Therefore, the sequence $\left(e_{n}\right)_{n \in \mathbf{N}}$ is Bourbaki-Cauchy (not Cauchy) in $\ell_{2}$ but it is not Bourbaki-Cauchy in itself. Moreover, this sequence is also cofinally Bourbaki-Cauchy in $\ell_{2}$ but it is not cofinally Cauchy.

\section{Bourbaki-completeness and cofinal Bourbaki-completeness}

In this section we will introduce the completeness properties in metric spaces associated to the new classes of sequences defined in the previous section. We will start with the Bourbaki-Cauchy sequences.

Definition 7. A metric space $(X, d)$ is said to be Bourbaki-complete if every Bourbaki-Cauchy sequence in $X$ clusters (i.e., it has some convergent subsequence).

It is clear that every Bourbaki-complete metric space is complete. And, in order to see that the converse is not true, it is enough to take again the Hilbert space $\ell_{2}$. Indeed, the standard basis is a Bourbaki-Cauchy sequence in $\ell_{2}$ that have not any convergent subsequence (see Example 6).

Moreover every compact metric space is Bourbaki-complete since, in particular, every sequence in a compact metric space clusters. So, as we announced in the introduction, we can say that Bourbaki-completeness is an intermediate property between compactness and completeness.

$$
\text { compact } \Longrightarrow \text { Bourbaki-complete } \Longrightarrow \text { complete }
$$

Next result gives some relationships among these three properties. 
Theorem 8. The following statements are equivalent for a metric space $(X, d)$ :

(1) $X$ is compact.

(2) $X$ is totally bounded and complete.

(3) $X$ is Bourbaki-bounded and Bourbaki-complete.

Proof. It is well known the equivalence between (1) and (2) (see for instance [34]). On the other hand, as we said before, if $X$ is compact then it is Bourbaki-complete. And since every compact space is totally bounded then it is also Bourbaki-bounded, and hence (1) implies (3). Conversely, in order to see that (3) imply (1), take any sequence of $X$. By Bourbaki-boundedness this sequence has a Bourbaki-Cauchy subsequence and by Bourbaki-completeness this subsequence clusters. Therefore every sequence in $X$ clusters and then $X$ is compact.

Another useful relation between compactness and Bourbaki-completeness is the following.

Theorem 9. A metric space is Bourbaki-complete if, and only if, the closure of every Bourbaki-bounded subset is compact.

Proof. Fist of all, note that the closure $\bar{B}$, of a Bourbaki-bounded set $B$ is also Bourbaki-bounded. Indeed, for every $\varepsilon>0$ there exist $m \in \mathbf{N}$ and some points $p_{1}, \ldots, p_{k} \in X$ such that $B \subset \bigcup_{i=1}^{k} B_{\varepsilon}^{m}\left(p_{i}\right)$. Since $\bar{B} \subset \bigcup_{i=1}^{k} B_{\varepsilon}^{m+1}\left(p_{i}\right)$, we follows that $\bar{B}$ is also Bourbaki-bounded in $X$. Now, let $(X, d)$ be Bourbaki-complete and $B$ a Bourbaki-bounded subset of $X$. In order to see that $\bar{B}$ is compact, let $\left(x_{n}\right)_{n \in \mathbf{N}}$ be a sequence of $\bar{B}$. Then, according to Theorem $4,\left(x_{n}\right)_{n \in \mathbf{N}}$ has a Bourbaki-Cauchy subsequence in $X$. Then by Bourbaki-completeness this subsequence clusters in $X$. But, $\bar{B}$ is closed and then $\left(x_{n}\right)_{n \in \mathbf{N}}$ clusters in $\bar{B}$. Therefore $\bar{B}$ is compact.

Conversely, let $\left(x_{n}\right)_{n \in \mathbf{N}}$ be a Bourbaki-Cauchy sequence of $X$ then $\left\{x_{n}: n \in \mathbf{N}\right\}$ is a Bourbaki-bounded subset of $X$, and by hypothesis $\overline{\left\{x_{n}: n \in \mathbf{N}\right\}}$ is compact. Hence $\left(x_{n}\right)_{n \in \mathbf{N}}$ clusters, and therefore $(X, d)$ is Bourbaki-complete.

Then last result says that a metric space is Bourbaki-complete if, and only if, every closed and Bourbaki-bounded subset is compact. According to this fact, we are going to see that only finite dimensional Banach spaces can be Bourbaki-complete. In particular, that means that in some sense completeness and Bourbaki-completeness are very far from one another. Recall that an analogous result exists for cofinal completeness (see Beer [5]).

Corollary 10. A Banach space is Bourbaki-complete if, and only if, it is finite dimensional.

Proof. It is clear that every finite dimensional Banach space is Bourbaki-complete since every closed and bounded subset is compact. Conversely, if the Banach space is a Bourbaki-complete metric space, then according last result, its unit closed ball must be compact since in normed spaces bounded subsets are also Bourbaki-bounded. Finally, if the unit ball of a normed space is compact, then it is well known that it must have finite dimension.

Next, we introduce the completeness notion associated to the cofinally BourbakiCauchy sequences.

Definition 11. A metric space is said to be cofinally Bourbaki-complete if every cofinally Bourbaki-Cauchy sequence clusters. 
It is clear that cofinal Bourbaki-completeness implies both Bourbaki-completeness and cofinal completeness. In order to see that the reverse implications do not hold, we will see that in fact there exist Bourbaki-complete metric spaces that are not cofinally complete (Example 15) and also cofinally complete metric spaces that are not Bourbaki-complete (Example 16).

Note that this new kind of completeness is again a property between compactness and completeness. Moreover, we can easily obtain an analogous result to Theorem 8 in this context, whose proof follows at once from Theorems 4 and 8.

Theorem 12. The following statements are equivalent for a metric space $(X, d)$ :

(1) $X$ is compact.

(2) $X$ is totally bounded and cofinally complete.

(3) $X$ is Bourbaki-bounded and cofinally Bourbaki-complete.

Recall that the equivalence between (1) and (2) was pointed by Beer in [5]. On the other hand, according to Theorem 8, it is clear that in above condition (3) cofinal Bourbaki-completeness should be paired with a weaker boundedness notion corresponding to the property that each sequence has a cofinally Bourbaki-Cauchy subsequence. But note that, this weaker notion would be again Bourbaki-boundedness, as we can deduce easily from Theorem 4.

Now, it is interesting to see that our completeness properties are not only weaker than compactness but also weaker that uniform local compactness. Recall that a metric space $(X, d)$ is said to be uniformly locally compact whenever there exists some $\delta>0$ such that the set $\overline{B_{\delta}(x)}$ is compact, for every $x \in X$.

Proposition 13. Every uniformly locally compact metric space is cofinally Bourbaki-complete.

Proof. Firstly, let $\delta>0$ such that, for every $x \in X, \overline{B_{\delta}(x)}$ is compact. We can see that if $K \subset X$ is compact, then $\overline{K^{\delta / 2}}$ is also compact. Indeed, from the open cover of $K \subset \bigcup_{y \in K} B_{\delta / 2}(y)$, we can take a finite subcover $K \subset \bigcup_{i=1}^{n} B_{\delta / 2}\left(y_{i}\right)$. Since

$$
K^{\delta / 2} \subset \bigcup_{i=1}^{n}\left(B_{\delta / 2}\left(y_{i}\right)\right)^{\delta / 2} \subset \bigcup_{i=1}^{n} \overline{B_{\delta}\left(y_{i}\right)}
$$

and $\bigcup_{i=1}^{n} \overline{B_{\delta}\left(y_{i}\right)}$ is compact, it follows that $\overline{K^{\delta / 2}}$ is compact, as we wanted. And that means that, in particular, for every $x \in X$ and every $m \in \mathbf{N}$, the set $\overline{B_{\delta / 2}^{m}(x)}$ is compact.

Now, if $\left(x_{n}\right)_{n \in \mathbf{N}}$ is a cofinally Bourbaki-Cauchy sequence, then there exist $x \in X$ and $m \in \mathbf{N}$ such that $\left\{x_{n}: n \in \mathbf{N}\right\}$ is cofinally contained in $\overline{B_{\delta / 2}^{m}(x)}$. Therefore, by compactness of $\overline{B_{\delta / 2}^{m}(x)}$, we have that $\left(x_{n}\right)_{n \in \mathbf{N}}$ clusters.

According to the above, we have the following diagram:

uniformly locally compact $\Longrightarrow$ cofinally Bourbaki-complete $\Longrightarrow$ cofinally complete

Now, from a result by Rice [30], we know that in the frame of cofinally complete metric spaces, the local compactness and the uniform local compactness are equivalent (see also Beer [5]). Then clearly the same happens for cofinally Bourbakicomplete metric spaces. In fact, we can improve slightly this result. For that, we need to introduce the next natural notions. Similarly to local compactness, we say 
that a metric space $(X, d)$ is locally Bourbaki-bounded (resp., locally totally bounded), if for every $x \in X$, there exists a neighborhood of $x$ which is a Bourbaki-bounded (resp., totally bounded) subset of $X$.

Theorem 14. For a metric space $(X, d)$ the following statements are equivalent:

(1) $X$ is uniformly locally compact.

(2) $X$ is locally totally bounded and cofinally complete.

(3) $X$ is locally Bourbaki-bounded and cofinally Bourbaki-complete.

Proof. That $(1) \Rightarrow(3)$ follows at once from Proposition 13.

$(3) \Rightarrow(2)$ This implication can be obtained easily taking into account that, in particular, condition (3) implies that $X$ is in addition locally compact. Indeed, let $x \in X$ and let $V$ be a Bourbaki-bounded neighborhood of $x$. Take $B$ any closed ball around $x$ contained in $V$. Now it is easy to check that $B$ is both Bourbakibounded (since this property is hereditary) and cofinally Bourbaki-complete (since this property is inherited by closed sets). Then, from Theorem $12, B$ is a compact neighborhood of $x$.

$(2) \Rightarrow(1)$ Firstly note that, as in the above implication, we can see that (2) implies also the local compactness of $X$. Next, suppose by contradiction that $X$ is not uniformly compact, then for every $n \in \mathbf{N}$, there exists $x_{n} \in X$ such that $\overline{B_{1 / n}\left(x_{n}\right)}$ is not compact. Then, by local compactness of $X$, we can assert that the sequence $\left(x_{n}\right)_{n \in \mathbf{N}}$ does not cluster. Now, for every $n \in \mathbf{N}$, let $\left(y_{k}^{n}\right)_{k \in \mathbf{N}}$ be a sequence in $\overline{B_{1 / n}\left(x_{n}\right)}$ without cluster points. Next, consider a partition of $\mathbf{N}$ into a countable family of infinite subsets $\left\{M_{n}, n \in \mathbf{N}\right\}$. Finally, defining the sequence $z_{k}=y_{k}^{n}$, if $k \in M_{n}$, it is easy to check that $\left(z_{k}\right)_{k \in \mathbf{N}}$ is a cofinally Cauchy sequence which does not cluster.

We present now two examples in order to clarify the relationships among the above properties.

Example 15. Let $\ell_{2}$ be the Hilbert space, and let $X \subset \ell_{2}$ the discrete subspace, $X=\bigcup_{n \in \mathbf{N}} A_{n}$, where

$$
A_{n}=\left\{e_{n}\right\} \cup\left\{e_{n}+\frac{1}{n} e_{k}: k \in \mathbf{N}\right\}
$$

and $\left\{e_{n}: n \in \mathbf{N}\right\}$ is the standard basis of $\ell_{2}$. Then $X$ is locally compact since, for every $n \in \mathbf{N}$, the open ball of radius $1 / n$ and center $e_{n}$ or $e_{n}+\frac{1}{n} e_{k}$ is just the center point. But it is not uniformly locally compact. Indeed, for every $n \in \mathbf{N}$, the closed ball of radius $1 / n$ and center $e_{n}$ is the infinite discrete space $A_{n}$ which is clearly not compact. On the other hand, $X$ is Bourbaki-complete because every Bourbaki-Cauchy sequence is eventually constant. Therefore, by Theorem $14, X$ is not cofinally Bourbaki-complete neither cofinally complete since it is not uniformly locally compact.

Example 16. For a infinite cardinal number $\mathfrak{m}$, let $X=J(\mathfrak{m})$ the hedgehog space of spininess $\mathfrak{m}$ (see for instance [15]). That is, for a set $S$ with cardinal $\mathfrak{m}$, the space $X$ is just the disjoint union $\bigcup_{s \in S}([0,1] \times\{s\})$, after identifying all the points of the form $(0, s)$ to one only point $\mathbf{0} \equiv[(0, s)]$, endowed with the metric:

$$
d\left(\left[\left(x, s_{1}\right)\right],\left[\left(y, s_{2}\right)\right]\right)=\left\{\begin{array}{lll}
|x-y| & \text { if } & s_{1}=s_{2}, \\
x+y & \text { if } & s_{1} \neq s_{2} .
\end{array}\right.
$$


It is easy to see that this space is complete and not compact (note that the infinite closed set $\{[(1, s)]: s \in S\}$ is discrete). Besides, $X$ is Bourbaki-bounded. In fact, for every $\varepsilon>0, X$ is contained in the set $B_{\varepsilon}^{m}(0)$, for $m>1 / \varepsilon$. Then, from Theorem $8, X$ is not Bourbaki-complete. We are going to prove that this space is cofinally complete. Let $\left(\left[\left(x_{n}, s_{n}\right)\right]\right)_{n \in \mathbf{N}}$ be a cofinally Cauchy sequence. Then for every $\varepsilon>0$ there exists $\mathbf{N}_{\varepsilon} \subset \mathbf{N}$ such that $d\left(\left[\left(x_{i}, s_{i}\right)\right],\left[\left(x_{j}, s_{j}\right)\right]\right)<\varepsilon$, for all $i, j \in \mathbf{N}_{\varepsilon}$. If for some $\varepsilon>0$ there exists an infinite subset $C \subset \mathbf{N}_{\varepsilon}$ such that for every $i, j \in C$, we have $s_{i}=s_{j}$, then $\left(\left[\left(x_{n}, s_{n}\right)\right]\right)_{n \in \mathbf{N}}$ clusters because any sequence in the Euclidean unit interval $[0,1]$ clusters. Otherwise, for every $k \in \mathbf{N}$, we can choose $\left[\left(x_{n_{k}}, s_{n_{k}}\right)\right]$ such that $\left.d\left(\left[\left(x_{n_{k}}, s_{n_{k}}\right)\right], \mathbf{0}\right)\right)<1 / k$, for some $n_{k} \in \mathbf{N}_{1 / k}$. Then, the subsequence $\left(\left[\left(x_{n_{k}}, s_{n_{k}}\right)\right]\right)_{k \in \mathbf{N}}$ converges to $\mathbf{0}$ and then the initial sequence clusters, as we wanted. In particular, we have seen that cofinal completeness does not imply Bourbaki-completeness as well as cofinal completeness does not imply cofinal Bourbaki-completeness.

We finish this section just linking Bourbaki and cofinal Bourbaki-completeness with the well known class of UC-spaces. In this line, we are going to see that every UC metric space is cofinally Bourbaki-complete, and hence Bourbaki-complete. Recall that a metric space $(X, d)$ is called $U C$ or Atsuji when every real continuous function on $X$ is uniformly continuous. There are several characterizations of these spaces, as we can see in the nice paper by Jain and Kundu [26]. In spite of there exists a sequential characterization of UC-spaces given by Toader in [33] that involves a Cauchy-like condition, we will use the characterization given by Hueber in terms the so-called isolation functional. Namely, if $I: X \rightarrow[0, \infty)$ is the functional defined by $I(x)=d(x, X-\{x\})$, then it is proved in [25] that a metric space is UC when every sequence $\left(x_{n}\right)_{n}$ with $\lim _{n \rightarrow \infty} I\left(x_{n}\right)=0$ clusters.

Proposition 17. Every UC metric space is cofinally Bourbaki-complete.

Proof. Let $\left(x_{n}\right)_{n}$ be a cofinally Bourbaki-Cauchy sequence, that we can suppose has no constant subsequence. According to the above characterization by Hueber, we are going to see that $\left(x_{n}\right)_{n}$ has a subsequence along which the isolation functional goes to zero. Indeed, for every $j \in \mathbf{N}$ there exist $m_{j} \in \mathbf{N}$ and $p_{j} \in X$ such that $\left\{x_{n}: n \in\right.$ $\mathbf{N}\}$ is cofinally contained in $B_{1 / j}^{m_{j}}\left(p_{j}\right)$. Since every $x \in B_{1 / j}^{m_{j}}\left(p_{j}\right)$ satisfies $I(x)<1 / j$, then we can construct a subsequence $\left(x_{n_{j}}\right)_{j}$ of $\left(x_{n}\right)_{n}$ such that $\lim _{j \rightarrow \infty} I\left(x_{n_{j}}\right)=0$, as we wanted.

In order to see that the converse of last result is not true, it is enough to consider the real line $\mathbf{R}$ with the usual metric.

\section{Bourbaki-completeness by nets}

Recall that a property which is given in terms of sequences in the frame of metric spaces, can be also reformulated in terms of nets and entourages in order to generalize it to the setting of uniform spaces. For example that happens when we define a uniform space to be complete when every Cauchy net clusters (converges). Then, we give the following definition.

Definition 18. A net $\left(x_{\lambda}\right)_{\lambda \in \Lambda}$ in a uniform space $(X, \mathbf{U})$, is said to be BourbakiCauchy in $X$ if for every entourage $U \in \mathbf{U}$ there exist $\lambda_{0} \in \Lambda$ and $m \in \mathbf{N}$ such that for some $x \in X, x_{\lambda} \in U^{m}[x]=U \circ \stackrel{(m)}{\circ} \circ U[x]$, for every $\lambda \geq \lambda_{0}$. 
Hence, in the case of a metric space $(X, d)$, a net $\left(x_{\lambda}\right)_{\lambda \in \Lambda}$ will be BourbakiCauchy in $X$ if, for every $\varepsilon>0$ there exist $m \in \mathbf{N}$ and $\lambda_{0} \in \Lambda$ such that for some $x \in X, x_{\lambda} \in B_{\varepsilon}^{m}(x)$, for every $\lambda \geq \lambda_{0}$.

Clearly, we can generalize the notion of Bourbaki-completeness for uniform spaces, saying that a uniform space is Bourbaki-complete if every Bourbaki-Cauchy net in it, clusters. We are not interested here in the study of this property in this general setting. In fact, such study will be done in a forthcoming paper which is still in preparation and where we will compare different kind of completeness in uniform spaces. For instance we will see there that Bourbaki-completeness of uniform spaces is equivalent to the so-called $\delta$-completeness introduced by García-Máynez in [16].

Now, we are going to prove the announced result saying that for metrizable spaces is equivalent to be Bourbaki-complete by sequences and by nets. As we mentioned in the introduction the following proof is due to Hohti and Junnila (University of Helsinki) and it has been communicated privately to the authors.

Theorem 19. Let $(X, d)$ be a metric space. Then $X$ is Bourbaki-complete if and only if every Bourbaki-Cauchy net in $X$ clusters.

Proof. We only need to prove sufficiency. Suppose that $\left(x_{\lambda}\right)_{\lambda \in \Lambda}$ is a BourbakiCauchy net that does not have a cluster point. Then each point $x \in X$ has an open neighborhood $V^{x}$ such that $\left(x_{\lambda}\right)_{\lambda \in \Lambda}$ is not cofinally in $V^{x}$. By paracompactness, the open cover $\mathcal{V}=\left\{V^{x}: x \in X\right\}$ has an open locally finite refinement $\mathcal{A}$, which is in particular point-finite. We will inductively construct a Bourbaki-Cauchy subsequence $\left(x_{n}\right)_{n \in \mathbf{N}}$ of $\left(x_{\lambda}\right)_{\lambda \in \Lambda}$ which does not have any cluster point.

Let $x_{0} \in X$ an arbitrary point. As $\mathcal{A}$ is point-finite, there are only finitely many $A_{1}, \ldots, A_{k} \in \mathcal{A}$ such that $x_{0} \in A_{i}, i=1, \ldots, k$. Let $\mathcal{A}_{0}$ denote the finite subfamily of $\mathcal{A}$ consisting of all $A_{i}, i=1, \ldots, k$. For each $A_{i},\left(x_{\lambda}\right)_{\lambda \in \Lambda}$ is residually in $X-A_{i}$, so it is residually in $X-\cup \mathcal{A}_{0}$. Thus, we can select some $\lambda_{1} \in \Lambda$ such that for every $\lambda \geq \lambda_{1}, x_{\lambda} \in X-\bigcup \mathcal{A}_{0}$ and $x_{\lambda} \in B_{1 / 2}^{m_{1}}\left(y_{1}\right)$, for some $y_{1} \in X$ and $m_{1} \in \mathbf{N}$. Now, take the finite subfamily $\mathcal{A}_{1}=\mathcal{A}_{0} \cup\left\{A \in \mathcal{A}: x_{\lambda_{1}} \in A\right\}$. The net $\left(x_{\lambda}\right)_{\lambda \in \Lambda}$ is again residually in $X-\cup \mathcal{A}_{1}$ and we can find some $\lambda_{2} \geq \lambda_{1}$ such that for every $\lambda \geq \lambda_{2}$, $x_{\lambda} \in X-\bigcup \mathcal{A}_{1}$ and $x_{\lambda} \in B_{1 / 2^{2}}^{m_{2}}\left(y_{2}\right)$, for some $y_{2} \in X$ and $m_{2} \in \mathbf{N}$. In general, for every $n \geq 3$, there is some $\lambda_{n} \geq \lambda_{n-1}$ such that for every $\lambda \geq \lambda_{n}, x_{\lambda} \in X-\bigcup \mathcal{A}_{n-1}$, where $\mathcal{A}_{n-1}=\mathcal{A}_{n-2} \cup\left\{A \in \mathcal{A}: x_{\lambda_{n-1}} \in A\right\}$ is a finite subfamily of $\mathcal{A}$ and $x_{\lambda} \in B_{1 / 2^{n}}^{m_{n}}\left(y_{n}\right)$, for some $y_{n} \in X$ and $m_{n} \in \mathbf{N}$. Note that the sequence $\left(x_{\lambda_{n}}\right)_{n \in \mathbf{N}}$ obtained in this way is Bourbaki-Cauchy. However, $\left(x_{\lambda_{n}}\right)_{n \in \mathbf{N}}$ does not have a cluster point. Indeed, for any $y \in X, V^{y}$ is a neighborhood of $y$ such that $\left(x_{\lambda_{n}}\right)_{n \in \mathbf{N}}$ is residually in $X-V^{y}$, because by the construction of the sequence, $x_{\lambda_{n}} \in V^{y}$ implies $x_{\lambda_{m}} \in X-V^{y}$, for all $m>n$.

\section{Bourbaki-completely metrizable spaces}

This section is devoted to study the (topological) problem of metrization by means of a Bourbaki-complete metric. Thus, we say that a metrizable space $X$ is Bourbaki-completely metrizable when there exists a Bourbaki-complete metric on $X$ giving its topology. We are interested here in obtaining a result in the line of the well known given by Čech ([12]) saying that a metrizable space $X$ is completely metrizable if and only if it is Cech complete, i.e., $X$ is a $G_{\delta^{-}}$set in its Stone-Cech compactification, $\beta X$. 
First of all we need to establish some properties about Bourbaki-completeness. Note that to be metrizable by a Bourbaki-complete metric is a topological property that is hereditary with respect to closed subsets. Moreover, as it happens to complete metrizability, we are going to prove that it is also a countable productive property. As a consequence of that, we will see that it would be also hereditary with respect to $G_{\delta}$-sets.

Theorem 20. Let $\left(X_{n}, d_{n}\right)_{n \in \mathbf{N}}$ be a countable family of metric spaces. Let $X=\prod_{n \in \mathbf{N}} X_{n}$ be the product space endowed with the metric

$$
\rho(x, y)=\sum_{n \in \mathbf{N}} \frac{d_{n}^{*}\left(x_{n}, y_{n}\right)}{2^{n}}
$$

where $d_{n}^{*}=\min \left\{d_{n}, 1\right\}$. Then, $(X, \rho)$ is Bourbaki-complete if, and only if, $\left(X_{n}, d_{n}\right)$ is Bourbaki-complete, for every $n \in \mathbf{N}$.

Proof. One implication is clear since every $\left(X_{n}, d_{n}\right)$ is homeomorphic to a closed subspace of $(X, \rho)$. Conversely, suppose that $\left(X_{n}, d_{n}\right)$ is Bourbaki-complete, for every $n \in \mathbf{N}$. Since the metrics $d_{n}$ and $d_{n}^{*}$ are uniformly equivalent, we have that $\left(X_{n}, d_{n}^{*}\right)$ is also a Bourbaki-complete metric space, for every $n \in \mathbf{N}$. Let $\left(x^{n}\right)_{n \in \mathbf{N}}$ be a Bourbaki-Cauchy sequence in $(X, \rho)$. Observe that, for every $i \in \mathbf{N}$, we get that $\left(x_{i}^{n}\right)_{n \in \mathbf{N}}$ is a Bourbaki-Cauchy sequence of $\left(X_{i}, d_{i}^{*}\right)$. Therefore for $i=1$, there exists a subsequence $\left(x_{1}^{n}\right)_{n \in \mathbf{N}_{1}}$ of $\left(x_{1}^{n}\right)_{n \in \mathbf{N}}, \mathbf{N}_{1} \subset \mathbf{N}$, which converges to some $y_{1} \in X_{1}$. Now, the sequence $\left(x_{2}^{n}\right)_{n \in \mathbf{N}_{1}}$ of $\left(X_{2}, d_{2}^{*}\right)$, is a Bourbaki-Cauchy subsequence of $\left(x_{2}^{n}\right)_{n \in \mathbf{N}}$ since every subsequence of a Bourbaki-Cauchy sequence is also Bourbaki-Cauchy. Again, by Bourbaki-completeness of $\left(X_{2}, d_{2}^{*}\right)$ there exists a subsequence $\left(x_{2}^{n}\right)_{n \in \mathbf{N}_{2}}$ of $\left(x_{2}^{n}\right)_{n \in \mathbf{N}_{1}}, \mathbf{N}_{2} \subset \mathbf{N}_{1}$, which converges to some $y_{2} \in X_{2}$. If we proceed in this way, for every $i \geq 2$, there exists a subsequence $\left(x_{i}^{n}\right)_{n \in \mathbf{N}_{i}}$ of $\left(x_{i}^{n}\right)_{n \in \mathbf{N}_{i-1}}, \mathbf{N}_{i} \subset \mathbf{N}_{i-1}$ which converges to some $y_{i} \in X_{i}$. Next, let $y=\left(y_{i}\right)_{i \in \mathbf{N}} \in X$, we are going to check that $y$ is a cluster point of the sequence $\left(x^{n}\right)_{n \in \mathbf{N}}$.

Indeed, let $\varepsilon>0$ and $N \in \mathbf{N}$ large enough to satisfy that $\sum_{N+1}^{\infty} \frac{1}{2^{i}}<\varepsilon / 2$. Since $y_{i}$ is a limit point of $\left(x_{i}^{n}\right)_{n \in \mathbf{N}_{i}}$, for every $i \in \mathbf{N}$, we can choose some $n_{\varepsilon}$ such that, for every $i=1, \ldots, N$,

$$
d_{i}^{*}\left(x_{i}^{n}, y_{i}\right)<\frac{\varepsilon \cdot 2^{i}}{2 N}
$$

when $n \in \mathbf{N}_{N}, n \geq n_{\varepsilon}$. Computing, we have that

$$
\rho\left(x^{n}, y\right)=\sum_{i \in \mathbf{N}} \frac{d_{i}^{*}\left(x_{i}^{n}, y_{i}\right)}{2^{i}}=\sum_{i=1}^{N} \frac{d_{i}^{*}\left(x_{i}^{n}, y_{i}\right)}{2^{i}}+\sum_{i=N+1}^{\infty} \frac{d_{i}^{*}\left(x_{i}^{n}, y_{i}\right)}{2^{i}}<N \cdot \frac{\varepsilon}{2 N}+\frac{\varepsilon}{2}=\varepsilon
$$

for every $n \in \mathbf{N}_{N}, n>n_{\varepsilon}$. Therefore for every $\varepsilon>0$ the sequence $\left(x^{n}\right)_{n \in \mathbf{N}}$ is cofinally contained in the neighborhood $B_{\varepsilon}(y)$ of $y$. Hence, $y$ is a cluster point for $\left(x^{n}\right)_{n \in \mathbf{N}}$.

As a consequence of last result we obtain the following.

Theorem 21. A $G_{\delta}$-set in a Bourbaki-complete metric space is Bourbaki-completely metrizable.

Proof. Firstly let $G$ be an open subspace of a Bourbaki-complete metric space $(X, d)$. Consider the real-valued continuous function given by $f(x)=1 / d(x, X-G)$, for $x \in G$. By continuity of $f$, the metric defined on $G$ by

$$
\rho(x, y)=d(x, y)+|f(x)-f(y)|
$$


is topologically equivalent to the metric $d$ on $G$. And it is clear that if a sequence $\left(x_{n}\right)_{n \in \mathbf{N}}$ is Bourbaki-Cauchy in $(G, \rho)$ then it is also Bourbaki-Cauchy in $(G, d)$. So, let $\left(x_{n}\right)_{n \in \mathbf{N}}$ be a Bourbaki-Cauchy sequence in $(G, \rho)$ and fix some $\varepsilon>0$. Then there exist $m, n_{0} \in \mathbf{N}$ such that $x_{n} \in B_{\varepsilon}^{m}\left(x_{n_{0}}\right)$, for every $n \geq n_{0}$. In particular we have, that

$$
\left|f\left(x_{n}\right)-f\left(x_{n_{0}}\right)\right|<\rho\left(x_{n}, x_{n_{0}}\right)<m \cdot \varepsilon
$$

And then $f\left(x_{n}\right)<m \cdot \varepsilon+f\left(x_{n_{0}}\right)$, for all $n \geq n_{0}$. Hence $d\left(x_{n}, X-G\right)>\frac{1}{m \cdot \varepsilon+f\left(x_{n_{0}}\right)}>0$, for every $n \geq n_{0}$. Thus, we can choose some $\lambda>0$ such that

$$
\left\{x_{n}: n \in \mathbf{N}\right\} \subset X_{\lambda}=\{x \in X: d(x, X-G) \geq \lambda\} \subset G .
$$

Since $X_{\lambda}$ is a closed subset of $(X, d)$, which is Bourbaki-complete, we have that the sequence $\left(x_{n}\right)_{n \in \mathbf{N}}$ clusters in $X_{\lambda}$ and hence in $G$. Consequently, $(G, \rho)$ is Bourbakicomplete and $G$ is Bourbaki-completely metrizable.

On the other hand, let $H$ be a $G_{\delta^{-}}$set in $X$, then $H=\bigcap_{n=1}^{\infty} G_{n}$ where $G_{n}$ is an open subset of $X$, for every $n \in \mathbf{N}$. According to the above paragraph, we have that every $G_{n}$ is completely-Bourbaki metrizable. Now, from Theorem 20, the product space $\prod_{n=1}^{\infty} G_{n}$ is also Bourbaki-completely metrizable. And we finish taking into account that $H$ is homeomorphic to a closed subspace of $\prod_{n=1}^{\infty} G_{n}$, namely the diagonal subspace of $\prod_{n=1}^{\infty} G_{n}$.

Next, let $\mathcal{P}_{0}(X)$ be the family of non-empty subset of $X$. We are going to define a couple of functionals on $\mathcal{P}_{0}(X)$ in order to obtain in this frame results of type Cantor's Theorem and Kuratowski's Theorem known for complete metric spaces (see [8]).

Firstly, we define $\gamma: \mathcal{P}_{0}(X) \rightarrow[0, \infty]$ by,

$$
\gamma(A)=\inf \left\{\varepsilon>0: A \subset B_{\varepsilon}^{m}(x) \text { for some } m \in \mathbf{N} \text { and } x \in X\right\} .
$$

Clearly $\gamma(A)=\infty$ if and only if $A$ is not bounded by the metric. In some sense $\gamma$ is a generalization of the radius (or diameter) of a set. The functional $\gamma$ satisfies the following properties:

(i) If $A \subset B$, then $\gamma(A) \leq \gamma(B)$.

(ii) $\gamma(\bar{A})=\gamma(A)$. In fact, for any $\varepsilon>0$ such that $A \subset B_{\varepsilon}^{m}(x)$, for some $m \in \mathbf{N}$ and $x \in X$, we have that $\bar{A} \subset B_{\varepsilon}^{m+1}(x)$.

(iii) $\gamma(A)=0$ if and only if $\mathrm{A}$ is a uniformly chainable subset of $X$.

Recall that $A$ is uniformly chainable in $X$ if for every $\varepsilon>0$ there exists $m \in \mathbf{N}$ such that each two points in $A$ can be joined by an $\varepsilon$-chain in $X$ of length at most $m$ (see Beer [4]).

Now let $\eta: \mathcal{P}_{0}(X) \rightarrow[0, \infty]$ defined by,

$\eta(A)=\inf \left\{\varepsilon>0: A \subset \bigcup_{i=1}^{k} B_{\varepsilon}^{m}\left(x_{i}\right)\right.$ for some $m \in \mathbf{N}$ and finite $\left.x_{i} \in X, i=1, \ldots, k\right\}$.

Again $\eta(A)=\infty$ if, and only, if $A$ is not bounded. Note that, in some sense, $\eta$ is a generalization of the so-called Hausdorff measure of non-compactness (see [8]) defined by,

$$
\alpha(A)=\inf \left\{\varepsilon>0: A \subset \bigcup_{i=1}^{k} B_{\varepsilon}\left(x_{i}\right) \text { for some finite } x_{i} \in X, i=1, \ldots, k\right\} .
$$


The functional $\eta$ satisfies the following properties:

(i) If $A \subset B$, then $\eta(A) \leq \eta(B)$.

(ii) $\eta(\bar{A})=\eta(A)$.

(iii) $\eta(A)=0$ if and only if $A$ is a Bourbaki-bounded subset of $X$.

(iv) $\eta(A \cup B)=\max \{\eta(A), \eta(B)\}$.

Thus, next result characterizes the Bourbaki-completeness in terms of the above functionals.

Theorem 22. For a metric space $(X, d)$ the following statements are equivalent:

(1) $X$ is Bourbaki-complete.

(2) For every decreasing sequence $\left(A_{n}\right)_{n \in \mathbf{N}}$ of non-empty closed subsets of $X$ which satisfies that $\lim _{n \rightarrow \infty} \eta\left(A_{n}\right)=0$, then $K=\bigcap_{n \in \mathbf{N}} A_{n}$ is a non-empty compact set.

(3) For every decreasing sequence $\left(A_{n}\right)_{n \in \mathbf{N}}$ of non-empty closed subsets of $X$ which satisfies that $\lim _{n \rightarrow \infty} \gamma\left(A_{n}\right)=0$, then $K=\bigcap_{n \in \mathbf{N}} A_{n}$ is a non-empty compact set.

Proof. $\quad(1) \Rightarrow(2)$ Assume that $(X, d)$ is Bourbaki-complete and, for every $n \in \mathbf{N}$, let $x_{n} \in A_{n}$. Clearly the set $\left\{x_{n}: n \in \mathbf{N}\right\}$ is a Bourbaki-bounded subset of $X$ and hence, from Theorem 4 , the sequence $\left(x_{n}\right)_{n \in \mathbf{N}}$ has a Bourbaki-Cauchy subsequence which clusters by Bourbaki-completeness. Then, $K=\bigcap_{n \in \mathbf{N}} A_{n} \supset$ $\bigcap_{n \in \mathbf{N}} \overline{\left\{x_{m}: m \geq n\right\}} \neq \emptyset$. Finally, applying Theorem 8, we follow that $K$ is compact. Indeed, $K$ is Bourbaki-bounded subset of $X$ since $\eta(K)=0$, and it is also Bourbaki-complete since $K$ is closed in $X$.

$(2) \Rightarrow(3)$ This is clear, because $\eta(A) \leq \gamma(A)$, for every $A \subset X$.

$(3) \Rightarrow(1)$ Let $\left(x_{n}\right)_{n \in \mathbf{N}}$ be a Bourbaki-Cauchy sequence in $X$ and define the decreasing sequence of non-empty closed sets, $A_{n}=\overline{\left\{x_{m}: m \geq n\right\}}$. Now, since $\gamma\left(A_{n}\right)=0$, for every $n \in \mathbf{N}$, condition (3) says that the set $K=\bigcap_{n \in \mathbf{N}} A_{n}$ is nonempty. And then we finish since $K$ is just the set of cluster points of the sequence $\left(x_{n}\right)_{n \in \mathbf{N}}$.

Now, we have all the ingredients to give the desired result of Bourbaki-complete metrization. We will use the well-known metrization theorem by Dugunji [14], saying that "If $X$ is a metrizable space and $\left\{\mathcal{A}_{n}: n \in \mathbf{N}\right\}$ is a family of open covers of $X$, then $X$ can be metrizable by a metric such that the family of its open balls of radius $1 / n,\left\{B_{1 / n}(x): x \in X\right\}$, refines $\mathcal{A}_{n}$, for all $n \in \mathbf{N}$."

Theorem 23. Let $X$ be a metrizable space. The following statements are equivalent:

(1) $X$ is Bourbaki-completely metrizable.

(2) $X=\bigcap_{n=1}^{\infty} G_{n}$ where each $G_{n}$ is an open and paracompact subspace of $\beta X$.

(3) There exists some compactification $c X$ of $X$ such that $X=\bigcap_{n=1}^{\infty} G_{n}$ where each $G_{n}$ is an open and paracompact subspace of $c X$.

Proof. $(1) \Rightarrow(2)$ Let $(X, d)$ be a Bourbaki-complete metric space. For $\varepsilon>0$, define the following equivalence relation: $x \sim_{\varepsilon} y$ if, and only if, $y \in B_{\varepsilon}^{\infty}(x)$. This equivalence relation determines a partition $\mathcal{P}_{\varepsilon}=\{[x]: x \in X\}$ of $X$, where clearly $[x]=B_{\varepsilon}^{\infty}(x)$ is a clopen subset of $X$. 
For every $i \in \mathbf{N}$ take only one $x \in X$ for each equivalence class $B_{1 / i}^{\infty}(x) \in \mathcal{P}_{1 / i}$, and let $V_{x, i}^{m}$ a cozero subset of $\beta X$ such that

$$
V_{x, i}^{m} \cap X=B_{1 / i}^{m}(x) .
$$

Note that, by density of $X$ in $\beta X$, we have $V_{x, i}^{m} \cap V_{y, i}^{n} \neq \emptyset$ if, and only if, $B_{1 / i}^{m}(x) \cap$ $B_{1 / i}^{n}(y) \neq \emptyset$. Thus, for every $i \in \mathbf{N}$, the family $\left\{B_{1 / i}^{m}(x): B_{1 / i}^{\infty}(x) \in \mathcal{P}_{1 / i}, m \in \mathbf{N}\right\}$ is a star-countable cover of $X$. Hence, for each $i \in \mathbf{N}$, we have that $\left\{V_{x, i}^{m}: B_{1 / i}^{\infty}(x) \in\right.$ $\left.\mathcal{P}_{1 / i}, m \in \mathbf{N}\right\}$ is a cozero star-countable family of $\beta X$ which cover $X$. For every $i \in \mathbf{N}$, let $G_{i}=\bigcup_{B_{1 / i}^{\infty}(x) \in \mathcal{P}_{1 / i}} \bigcup_{m \in \mathbf{N}} V_{x, i}^{m}$. Recall that countable unions of cozero sets are cozero sets too, hence for $x \neq y$ (i.e., $[x] \neq[y]$ ), we have that $\bigcup_{m \in \mathbf{N}} V_{x, i}^{m}$ and $\bigcup_{m \in \mathbf{N}} V_{y, i}^{m}$ are disjoint cozero sets. Then every $G_{i}$ is a free union of open $F_{\sigma}$-sets of $\beta X$, that is, a free union of open Lindelöf subspaces of $\beta X$. Consequently, every $G_{i}$ is open and paracompact, since every regular Lindelöf space is paracompact and a free union of paracompact spaces is also paracompact.

Trivially $X \subset \bigcap_{i=1}^{n} G_{i}$. We have to prove the reverse inclusion. Suppose there is some $z \in \bigcap_{i=1}^{\infty} G_{i}-X$. Thus, for every $i \in \mathbf{N}$, there exists some $x_{i} \in X$ such that $z \in \bigcup_{m \in \mathbf{N}} V_{x_{i}, i}^{m}$. Hence for every $i \in \mathbf{N}$ there exists some $m_{i} \in \mathbf{N}$ such that $z \in V_{x_{i}, i}^{m_{i}}$ and clearly $V_{x_{i}, i}^{m_{i}}$ is a neighborhood of $z$ in $\beta X$. Let us write $V_{x_{i}, i}^{m_{i}}=U_{i}$. Thus, $\left\{U_{i}\right\}_{i \in \mathbf{N}}$ is a sequence of neighborhoods of $z$ that, without loss of generality we can suppose decreasing and with the property that $\gamma\left(U_{i} \cap X\right) \leq \frac{1}{i}$. Now, we can apply Theorem 22 to the sequence of closed sets,

$$
{\overline{U_{1} \cap X}}^{X} \supset{\overline{U_{2} \cap X}}^{X} \supset \ldots \supset{\overline{U_{i} \cap X}}^{X} \supset \ldots
$$

and then $K=\bigcap_{i \in \mathbf{N}}{\overline{U_{i} \cap X^{X}}}^{X}$ is a non-empty compact set in $X$. By regularity of $\beta X$ there exists a closed neighborhood $W^{z}$ of $z$ such that $W^{z} \cap K=\emptyset$. But, by

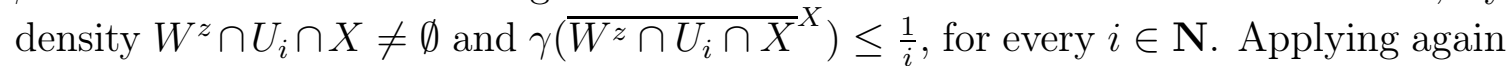
Theorem 22, it follows that

$$
W^{z} \cap K=W^{z} \cap\left(\bigcap_{i \in \mathbf{N}}{\overline{U_{i} \cap X}}^{X}\right)=\bigcap_{i \in \mathbf{N}} W^{z} \cap{\overline{\left(U_{i} \cap X\right)}}^{X} \supset \bigcap_{i \in \mathbf{N}}{\overline{W^{z} \cap U_{i} \cap X}}^{X} \neq \emptyset
$$

which is a contradiction. Therefore $z \in X$.

$(2) \Rightarrow(3)$ is trivial.

$(3) \Rightarrow(1)$ Let $X=\bigcap_{n=1}^{\infty} G_{n}$ where each $G_{n}$ is an open and paracompact subspace of $c X$. By regularity of $c X$ and for every $z \in G_{n}$, there exists an open neighborhood $U_{n}^{z}$ of $z$ in $c X$ such that

$$
z \in U_{n}^{z} \subset{\overline{U_{n}^{z}}}^{c X} \subset G_{n} .
$$

Then $\mathcal{U}_{n}=\left\{U_{n}^{z}: z \in G_{n}\right\}$ is an open cover of $G_{n}$. Since $G_{n}$ is paracompact let $\mathcal{V}_{n}$ be an open locally finite refinement of $\mathcal{U}_{n}$. For every $n \in \mathbf{N}$, let

$$
\mathcal{A}_{n}=\left\{V \cap X: V \in \mathcal{V}_{n}\right\} .
$$

Then $\mathcal{A}=\left\{\mathcal{A}_{n}: n \in \mathbf{N}\right\}$ is a countable family of open covers of $X$. Since $X$ is metrizable, by the Dugundji's theorem, there exists an admissible metric $d$ such that $\left\{B_{1 / n}(x): x \in X\right\}$ refines $\mathcal{A}_{n}$, for every $n \in \mathbf{N}$.

We are going to prove that $(X, d)$ is Bourbaki-complete. Let $\left(x_{n}\right)_{n \in \mathbf{N}}$ be a Bourbaki-Cauchy sequence in $(X, d)$. Then $\left(x_{n}\right)_{n \in \mathbf{N}}$ clusters in $c X$ by compactness. 
Thus, let

$$
z \in \bigcap_{n \in \mathbf{N}}{\overline{\left\{x_{j}: j \geq n\right\}}}^{c X} .
$$

Now, for every $i \in \mathbf{N}$, there exist $n_{0} \in \mathbf{N}$ and $m \in \mathbf{N}$ such that for some $x \in X$, $x_{n} \in B_{1 / i}^{m}(x)$ for every $n \geq n_{0}$. Since $B_{1 / i}^{m}(x)=\bigcup\left\{B_{1 / i}(y): y \in B_{1 / i}^{m-1}(x)\right\}$, then

$$
z \in{\overline{\left\{x_{n}: n \geq n_{0}\right\}}}^{c X} \subset{\overline{B_{1 / i}^{m}(x)}}^{c X} \subset{\overline{\bigcup\left\{V_{y, i}: y \in B_{1 / i}^{m-1}(x)\right\}}}^{c X}
$$

where $B_{1 / i}(y) \subset V_{y, i}$, for some $V_{y, i} \in \mathcal{V}_{i}$. Since $\mathcal{V}_{i}$ is a locally finite family sets of $c X$ then

$$
\begin{aligned}
z \in{\overline{\bigcup\left\{V_{y, i}: y \in B_{1 / i}^{m-1}(x)\right\}^{c}}}^{c X} & =\bigcup\left\{{\overline{V_{y, i}}}^{c X}: y \in B_{1 / i}^{m-1}(x)\right\} \\
& \subset \bigcup\left\{{\overline{U_{y, i}}}^{c X}: y \in B_{1 / i}^{m-1}(x)\right\} \subset G_{i}
\end{aligned}
$$

where $V_{y, i} \subset U_{y, i}$, for some $U_{y, i} \in \mathcal{U}_{i}$. Consequently, $z \in G_{i}$ for every $i \in \mathbf{N}$ and hence $z \in X$, as we wanted.

As an easy consequence of last result, we have that every locally compact metric space is Bourbaki-completely metrizable, since in fact it is open and paracompact in every of its compactifications. Moreover, according to Theorem 20, every metric space homeomorphic to a closed subset of a countable product of locally compact metric spaces will be also Bourbaki-completely metrizable. This is the case, for example, of $\mathbf{N}^{\mathbf{N}}$ or $\mathbf{R}^{\mathbf{N}}$.

It is clear that if a metric space is not completely metrizable (for instance, the rational numbers with the usual metric) then it cannot be Bourbaki-completely metrizable. The next example shows that, assuming the existence of measurable cardinals, not every completely metrizable space is Bourbaki-completely metrizable.

Example 24. For a measurable cardinal number $\mathfrak{m}$, let $X=J(\mathfrak{m})$ be the hedgehog space of spininess $\mathfrak{m}$ (see Example 16). Then we know that $X$ is a complete metric space. On the other hand, Comfort and Negrepontis in [11] prove that for every open paracompact subspace $Y$ such that $J(\mathfrak{m}) \subset Y \subset \beta J(\mathfrak{m})$, the realcompactification $v J(\mathfrak{m})$ satisfy that $J(\mathfrak{m}) \subset v J(\mathfrak{m}) \subset Y$. Therefore, since $X$ is not realcompact, then it cannot be the (countable) intersection of open paracompact subspaces of $\beta X$.

Recall that a metrizable space $X$ is completely metrizable if, and only if, it is a $G_{\delta}$-set in every compactification $c X$ (see [15]). However, from the above theorem we cannot assure that if $X=\bigcap_{n=1}^{\infty} G_{n}$, where $G_{n}$ are open paracompact subspaces of some compactification $c X$, then the same is true in every compactification of $X$. If we carefully review the proof of last Theorem 23, we can see that the StoneČech compactification in condition (2) can be replaced by any compactification of $X$ where $X$ is $z$-embedded. We say that $X$ is $z$-embedded in $Y$ when $X \subset Y$ and every zero-set in $X$ is the intersection with $X$ of a zero-set in $Y$. On the other hand, it is well known that every metric space $(X, d)$ is $z$-embedded in its Samuel compactification $s_{d} X$. In fact, as Woods proves in [35], $s_{d} X$ is characterized uniquely (up to equivalence) as the compactification where for every closed subset $A$ of $X$, the function $g_{A}(x)=\min \{d(x, A), 1\}$ extends continuously to $g_{A}^{*} \in C\left(s_{d} X\right)$ and $\bar{A}^{s_{d} X}=Z\left(g_{A}^{*}\right)$. So, we can assert that $X$ is completely-Bourbaki metrizable if, and 
only if, $X$ is a countable intersection of open paracompact subspaces of $c X$, for every compactification $c X \geq s_{d} X$. But the question is: what happens with the rest of compactifications of $X$ ? We do not know the general answer. But, in this line, we can say that it is possible for a metric space $(X, d)$ to find a compactification $c X$ satisfying last condition (3) and with $c X \leq s_{d} X$. For instance, if $(X, d)$ is a metric space locally compact but not separable (for example, $\mathbf{R}$ endowed with the discrete metric), then $X$ is open and paracompact in any compactification, and in particular in its one-point compactification $X^{\infty}$. But, in this case $X^{\infty}<s_{d} X$ since, according to a result by Woods in [35], for a locally compact metrizable space $X$, there exists some admissible metric $d$ such that the one point compactification of $X$ is equivalent to $s_{d} X$ if, and only if, $X$ is second countable.

At this point, it is interesting to take into account the class of $\delta$-complete topological spaces introduced and studied by García-Máynez in [16] (see also [18] and [17]). These spaces, considered in the general frame of uniform spaces, can be characterized as those spaces $X$ that are the intersection of all the open and paracompact subsets of $\beta X$ containing it. Then, it is clear that every Bourbaki-completely metrizable space is $\delta$-complete. We do not know whether or not every completely metrizable space $X$ which is also $\delta$-complete is Bourbaki-completely metrizable. Note that, for instance, the space $X$ in Example 24 is not $\delta$-complete neither.

We finish this section, by linking Bourbaki-complete metrizability with other properties in the context of metrizable spaces. Recall that a metrizable space $X$ is said to be strongly metrizable if, and only if, $X$ has a base which is the union of countably many star-finite open covers, or equivalently $X$ can be embedded into a space of the form $\kappa^{\mathbf{N}} \times[0,1]^{\mathbf{N}}$, where we consider the cardinal $\kappa$ endowed with the discrete metric (see for instance Pears [29]).

The following topological notion, as well as its uniform version, will be interesting in what follows.

Definition 25. A topological space $X$ is strongly paracompact if every open cover of $X$ has an open star-finite refinement.

It is known that every regular Lindelöf space is strongly paracompact, that every metrizable strongly paracompact space is strongly metrizable and that every strongly metrizable space is paracompact. There exist examples of metric spaces which do not satisfy the reverse implications. Thus, the product space $\aleph_{1}^{\mathbf{N}} \times[0,1]^{\mathbf{N}}$, where $\aleph_{1}$ is the first uncountable cardinal, is a strongly paracompact metric space which is not separable. On the other hand, $\aleph_{1}^{\mathbf{N}} \times(0,1)$ is strongly metrizable but not strongly paracompact. Finally the metric hedgehog $J\left(\aleph_{1}\right)$ is paracompact but not strongly metrizable [29].

Theorem 26. Every strongly paracompact (more generally, every strongly metrizable) completely metrizable space is Bourbaki-completely metrizable.

Proof. Suppose the general case where $X$ is strongly metrizable and completely metrizable. Then $X$ is a subspace of the Bourbaki-completely metrizable space $\kappa^{\mathbf{N}} \times$ $[0,1]^{\mathbf{N}}$, for some cardinal $\kappa$. Since Bourbaki-complete metrizability is hereditary with respect to $G_{\delta^{-}}$sets, then every space homeomorphic to a $G_{\delta^{-}}$-subset of $\kappa^{\mathbf{N}} \times$ $[0,1]^{\mathbf{N}}$ is Bourbaki-completely metrizable. But finally recall that a metrizable space is completely metrizable if, and only if, it is a $G_{\delta^{-}}$set in every metric embedding (see [11]). 
Note that from last result, every separable completely metrizable space is Bourbaki-completely metrizable, since in particular it is strongly paracompact. Thus, every separable infinite dimensional Banach space as well as the metric hedgehog of countable many spininess $J\left(\aleph_{0}\right)$ are examples of non Bourbaki-complete spaces that can be metrized by an admissible Bourbaki-complete metric. We do not know if every Bourbaki-complete metric space is strongly metrizable or not. Note that, for every (non-measurable) uncountable cardinal $\mathfrak{m}, J(\mathfrak{m})$ is not strongly metrizable, so we ask if in this case $J(\mathfrak{m})$ is Bourbaki-completely metrizable. Finally, note that Bourbaki-completely metrizable together with strongly metrizable does not implies strongly paracompact. For that consider again $X=\aleph_{1}^{\mathbf{N}} \times(0,1)$.

\section{Cofinally Bourbaki-completely metrizable spaces}

This last section will be devoted to obtain more properties of the cofinally Bourbaki-complete metric spaces, as well as to study when a metrizable space admits an equivalent cofinal Bourbaki-complete metric, that is, when it is cofinally Bourbakicompletely metrizable. Recall that the analogous problem for cofinal completeness of metrizable spaces was solved by Romaguera in [31]. He obtains there, for a metrizable space $X$, the equivalence between the cofinal complete metrizability and the compactness of the set

$$
n l c(X)=\{x \in X: x \text { has no compact neighborhood in } X\} .
$$

Next, we are going to give a characterization of cofinal Bourbaki-completeness in metric spaces by means of the above mentioned set $n l c(X)$, and with the help of the functional $\bar{\nu}: \mathcal{P}_{0}(X) \rightarrow[0, \infty]$ defined by

$$
\bar{\nu}(A)=\sup \{\nu(x): x \in A\} .
$$

where $\nu(x)=\sup \left\{\varepsilon>0: \overline{B_{\varepsilon}(x)}\right.$ is compact $\}$ if $x$ has a compact neighborhood, and $\nu(x)=0$, otherwise (see [5]). Moreover, we will see that cofinal Bourbakicompleteness by sequences are equivalent to the corresponding notion for nets. For that, we will use the fact that for cofinal completeness a result like that is true as we mentioned in the introduction (Beer [5]).

Definition 27. A net $\left(x_{\lambda}\right)_{\lambda \in \Lambda}$ in a uniform space $(X, \mathbf{U})$, is said to be cofinally Bourbaki-Cauchy in $X$ if for every entourage $U \in \mathbf{U}$ there exists $m \in \mathbf{N}$ such that for some $x \in X,\left(x_{\lambda}\right)_{\lambda \in \Lambda}$ is cofinally contained in $U^{m}[x]=U \circ \stackrel{(m)}{.} \circ U[x]$.

Thus, when the uniform space is a metric space $(X, d)$, a net $\left(x_{\lambda}\right)_{\lambda \in \Lambda}$ will be cofinally Bourbaki-Cauchy in $X$ if for every $\varepsilon>0$ there exists $m \in \mathbf{N}$, such that for some $x \in X,\left(x_{\lambda}\right)_{\lambda \in \Lambda}$ is cofinally contained in $B_{\varepsilon}^{m}(x)$.

Next result also contains some conditions for a complete or a cofinally complete space to be cofinally Bourbaki-complete.

Theorem 28. For a metric space $(X, d)$ the following statements are equivalent:

(1) $X$ is cofinally Bourbaki-complete.

(2) $X$ is cofinally complete and, for every $\varepsilon>0$ there exists $\delta>0$ such that $\gamma(A)<\delta$ implies $\alpha(A)<\varepsilon$, for $A \in \mathcal{P}_{0}(X)$.

(3) $X$ is complete and, for every $\varepsilon>0$ there is $\delta>0$ such that $\max \{\bar{\nu}(A), \gamma(A)\}<$ $\delta$ implies $\alpha(A)<\varepsilon$, for $A \in \mathcal{P}_{0}(X)$. 
(4) Either $X$ is uniformly locally compact or nlc $(X)$ is a non-empty compact set such that, for every $\varepsilon>0, X-n l c(X)^{\varepsilon}$ is uniformly locally compact in its relative topology, and there exists $\delta>0$ with $\alpha\left(B_{\delta}^{n}(x)\right)<\varepsilon$, whenever $x \in n l c(X), n \in \mathbf{N}$.

(5) Every cofinally Bourbaki-Cauchy net clusters.

Proof. $(1) \Rightarrow(2)$ Since every cofinally Bourbaki-complete metric space is cofinally complete, only the continuity condition in (2) is necessary to prove. Suppose, by contradiction, that there is some $\varepsilon_{0}>0$ such that for every $\delta>0$ there exists $A_{\delta} \in \mathcal{P}_{0}(X)$ such that $\gamma\left(A_{\delta}\right)<\delta$ but $\alpha\left(A_{\delta}\right) \geq \varepsilon_{0}$. In particular, for every $n \in \mathbf{N}$, it is possible to find a countable subset $\left\{x_{j}^{n}: j \in \mathbf{N}\right\}$ of $A_{1 / n}$ such that it is $\varepsilon_{0^{-}}$ discrete. Now, consider a partition of $\mathbf{N}$ into a countable family of infinite subsets $\left\{M_{n}, n \in \mathbf{N}\right\}$, and define the sequence $y_{j}=x_{j}^{n}$ if $j \in M_{n}$. Then $\left(y_{j}\right)_{j \in \mathbf{N}}$ is a cofinally Bourbaki-Cauchy sequence of $X$ which does not cluster.

$(2) \Leftrightarrow(3)$ This equivalence follows at once using the result by Beer in [5], saying that a complete metric space is cofinally complete if, and only if, for every $\varepsilon>0$ there exists $\delta>0$ such that for every $A \in \mathcal{P}_{0}$, if $\bar{\nu}(A)<\delta$ then $\alpha(A)<\varepsilon$.

$(2) \Rightarrow(4)$ Firstly, note that condition (2) implies that, for every $\varepsilon>0$ there exists $\delta>0$ such that, for every $x \in X$ and $n \in \mathbf{N}$, we have $\alpha\left(B_{\delta / 2}^{n}(x)\right)<\varepsilon$, since $\gamma\left(B_{\delta / 2}^{n}(x)\right) \leq \delta / 2<\delta$. On the other hand, if $n l c(X)=\emptyset$ then $X$ is locally compact and by cofinal completeness it is uniformly locally compact (see Theorem 14). The rest of condition (4) follows immediately from the characterization of cofinal completeness given by Beer in [5], saying that a metric space is cofinally complete if, and only if, $n l c(X)$ is compact and for every $\varepsilon>0$, the set $X-n l c(X)^{\varepsilon}$ is uniformly locally compact in its relative topology.

$(4) \Rightarrow(2)$ If $X$ is uniformly locally compact, we already know that $X$ is cofinally complete. Moreover, let $\delta>0$ be such that $\overline{B_{\delta}(x)}$ is compact for every $x \in X$. Then, it is easy to check that $\overline{B_{\delta / 2}^{n}(x)}$ is also compact, for every $n \in \mathbf{N}$ and $x \in X$ (see the proof of Theorem 13). Hence, for every $\varepsilon>0$ and $A \in \mathcal{P}_{0}(X)$ with $\gamma(A)<\delta / 2$, we have that $A$ must be totally bounded and then $\alpha(A)=0<\varepsilon$.

Now suppose that $n l c(X) \neq \emptyset$. Using again the result by Beer mentioned in the above implication, it is clear that condition (4) implies the cofinal completeness of $X$. Now let $\varepsilon>0$ and $x \in X$. If there exists $\delta>0$ such that $x \in B_{\delta}^{m}(y)$ for some $y \in n l c(X)$ and some $m \in \mathbf{N}$, then trivially $\alpha\left(B_{\delta}^{n}(x)\right)<\varepsilon$ for every $n \in \mathbf{N}$. Otherwise, suppose that $x \in X-B_{\delta}^{\infty}(y)$ for every $y \in n l c(X)$. Then $B_{\delta}^{n}(x) \subset X-n l c(X)^{\delta}$ for every $n \in \mathbf{N}$. Since $X-n l c(X)^{\delta}$ is uniformly locally compact in its relative topology there exists $\xi>0$ such that $\frac{B_{\xi}(z) \cap\left(X-n l c(X)^{\delta}\right)}{X-n l c(X)^{\delta}}$ is compact, for every $z \in X-n l c(X)^{\delta}$. Let $\nu<\min \{\delta, \xi\}$. Then, using the same argument as before, we have that

$$
{\overline{B_{\nu / 2}^{n}(x) \cap\left(X-n l c(X)^{\delta}\right)}}^{X-n l c(X)^{\delta}}={\overline{B_{\nu / 2}^{n}(x)}}^{X-n l c(X)^{\delta}}
$$

is also compact, for every $n \in \mathbf{N}$. Hence $\alpha\left(B_{\nu / 2}^{n}(x)\right)<\varepsilon$, as we wanted.

$(2) \Rightarrow(5)$ Let $\left(x_{\lambda}\right)_{\lambda \in \Lambda}$ be a cofinally Bourbaki-Cauchy net. From the hypothesis, for every $\varepsilon>0$ there exists $\delta>0$ such that if $x \in X$, we have $B_{\delta / 2}^{n}(x) \subset B_{\varepsilon}\left(x_{1}\right) \cup$ $\ldots \cup B_{\varepsilon}\left(x_{k}\right)$, for every $n \in \mathbf{N}$ and some points $x_{i} \in X, i=1, \ldots, k$. By definition of cofinally Bourbaki-Cauchy net, there exist $x \in X$ and $n \in \mathbf{N}$ such that $\left(x_{\lambda}\right)_{\lambda \in \Lambda}$ is cofinally contained in $B_{\delta / 2}^{n}(x)$. Hence $\left(x_{\lambda}\right)_{\lambda \in \Lambda}$ is cofinally contained in $B_{\varepsilon}\left(x_{j}\right)$, 
for some $j \in\{1, \ldots, k\}$. We have just proved that, in this case, every cofinally Bourbaki-Cauchy net is in fact cofinally Cauchy. Hence, since cofinal completeness by sequences and nets are equivalent in metric spaces (Beer [5]), we deduce that this net clusters.

$(5) \Rightarrow(1)$ It is clear.

The property of continuity type in the above condition (2) was also suggested by Hohti and Junnila. Note that it is equivalent to say that for all $\varepsilon>0$ there exists $\delta>0$ such that for every $x \in X$ and $n \in \mathbf{N}$ we have $\alpha\left(B_{\delta}^{n}(x)\right)<\varepsilon$. In order to see that cofinal completeness together with Bourbaki-completeness is not equivalent to cofinal Bourbaki-completeness, an example of cofinally complete and Bourbakicomplete metric space which does not satisfy the previously mentioned property would be needed. But until now we have not succeeded to find such a space. On the other hand, if we drop the completeness in condition (3), we have a necessary and sufficient condition for a metric space to have a cofinally Bourbaki-complete completion. Note, that the completion of a metric space $(X, d)$ is Bourbaki-complete if, and only if, every Bourbaki-bounded subset of $X$ is totally bounded. Finally, a geometric characterization of cofinal Bourbaki-completeness nicer than (4) would be better.

Contrary to what happens with Bourbaki-completeness, cofinal Bourbaki-completeness is not countably, neither finitely, productive. In fact we have the following result that is analogous to the obtained by Hohti in [22] for uniformly paracompact metric spaces. Recall that, as we mentioned in the introduction, cofinal completeness was shown to be equivalent to the notion of uniform paracompactness defined by Rice in [30].

Theorem 29. Let $(X, d)$ and $(Y, \rho)$ two cofinally Bourbaki-complete metric space. Then $X \times Y$ is cofinally Bourbaki-complete if and only if one of the following conditions holds:

(1) Either $X$ or $Y$ is compact.

(2) Both $X$ and $Y$ are locally compact.

Proof. The proof follows using the analogous result by Hohti [22] for uniform paracompactness (i.e., for cofinal completeness), together with our last Theorem 28. Indeed, the necessary condition is clear. For the sufficiency, suppose first that both spaces are locally compact. Since, by the hypothesis, they are cofinally complete then they must be uniformly locally compact (Theorem 14). In this case $X \times Y$ is also uniformly locally compact and, by Theorem 13, it is cofinal Bourbaki-complete.

Suppose now that $Y$ is compact and $X$ is cofinally Bourbaki-complete but not locally compact. We are going to apply condition (2) of last theorem. Note that it is only necessary to check the continuity property in (2), or equivalently that for all $\varepsilon>0$ there exists $\delta>0$ such that for every $(x, y) \in X \times Y$ and $n \in \mathbf{N}$ we have $\alpha\left(B_{\delta}^{n}(x, y)\right)<\varepsilon$. Indeed, let $\varepsilon>0$. By compactness of $Y$, for every $\delta>0$ and $y \in Y$ we have that $\alpha\left(B_{\delta}^{n}(y)\right)=0<\varepsilon$, for every $n \in \mathbf{N}$. Since $X$ is cofinally Bourbaki-complete, given $\varepsilon>0$ there is $\delta_{\varepsilon}>0$ such that for every $x \in X$ and $n \in \mathbf{N}$ we have that $\alpha\left(B_{\delta_{\varepsilon}}^{n}(x)\right)<\varepsilon$. And then for every $(x, y) \in X \times Y$ and $n \in \mathbf{N}$ we have that $\alpha\left(B_{\delta_{\varepsilon}}^{n}(x, y)\right) \stackrel{\leq}{\leq} \alpha\left(B_{\delta_{\varepsilon}}^{n}(x)\right)<\varepsilon$.

Now, and before studying the problem of the cofinal Bourbaki-complete metrization, we are going to see the relationships between this property and some other of 
uniform paracompactness type. At this point, we think that a uniform version of the strong paracompactness will be useful for our purposes, since cofinal completeness is equivalent to uniform paracompactness and, as we have seen in Theorem 26, strong paracompactness implies Bourbaki-completeness. Indeed, we are going to see that now we can obtain not only some implication but in fact that cofinal Bourbakicompleteness and uniformly strong paracompactness are equivalent (Theorem 32 below). We will do that in the setting of metric spaces but the same works for uniform spaces. Next definition is the metric version of the uniform notion given by Hohti in [22] under the name of uniform hypocompactness. Note that there is another definition of uniform strong paracompactness that was given by Musaev in [27]. In spite of they are different from the point of view of uniform spaces, they are nevertheless topologically equivalent.

Definition 30. A metric space $(X, d)$ is uniformly strongly paracompact if for every open cover $\mathcal{G}$ of $X$ there exists an open refinement $\mathcal{A}$ for which there is some $\delta>0$ such that, for every $A \in \mathcal{A}$, the set $A^{\delta}$ meets at most finitely many $A^{\prime} \in \mathcal{A}$. Such a family $\mathcal{A}$ is said to be an open uniformly star-finite refinement of $\mathcal{G}$.

For a family $\mathcal{G}$ we denote by $\mathcal{G}_{f}$ the family of finite unions of elements in $\mathcal{G}$. Recall that a cover $\mathcal{G}$ of $X$ is called directed when $\mathcal{G}_{f}$ refines $\mathcal{G}$. A cover $\mathcal{G}$ of a metric space $(X, d)$ is said to be uniformly directed if there is some $\delta>0$ such that the cover $\mathcal{G}_{f}^{\delta}$ refines $\mathcal{G}$, where $\mathcal{G}_{f}^{\delta}=\left\{H^{\delta}: H \in \mathcal{G}_{f}\right\}$.

In order to obtain one of the main results in this section, we will use the following metric version of a theorem given by Hohti in [22] for uniform spaces.

Theorem 31. (Hohti [22]) A metric space $(X, d)$ is uniformly strongly paracompact if and only if every directed open cover of $X$ has a uniformly directed refinement.

Theorem 32. A metric space is uniformly strongly paracompact if and only if it is cofinally Bourbaki-complete.

Proof. First, suppose $(X, d)$ is a cofinally Bourbaki-complete metric space, and let $\mathcal{G}=\left\{G_{\lambda}: \lambda \in \Lambda\right\}$ be a directed open cover of $X$. If $X=G_{\lambda}$ for some $\lambda \in \Lambda$ then $\mathcal{G}$ is a uniformly directed refinement of itself. So assume that $F_{\lambda}=X-G_{\lambda} \neq \emptyset$ for each $\lambda \in \Lambda$. Put $E=\left\{\left(F_{\lambda}, x\right): \lambda \in \Lambda, x \in F_{\lambda}\right\}$ and for each $\lambda$ let $<_{\lambda}$ be a well ordering of $F_{\lambda}$. Then $(E,<)$ is a directed set where $<$ is defined on $E$ by $\left(F_{\lambda}, x\right)<\left(F_{\nu}, y\right)$ if $\lambda \neq \nu$ then $F_{\lambda} \supseteq F_{\nu}$ or if $\lambda=\nu$ then $x<_{\lambda} y$. For each $\left(F_{\lambda}, x\right)$ put $\psi\left(F_{\lambda}, x\right)=x$. Then $\psi: E \rightarrow X$ is a net in $X$. Since $\mathcal{G}$ is a cover of $X, \psi$ cannot cluster in $X$, so $\psi$ cannot be cofinally Bourbaki-Cauchy. Therefore, there exists $\varepsilon>0$ such that if $y \in X$ then for every $m \in \mathbf{N}$ there exists $\left(F_{\lambda_{0}}, x_{0}\right) \in E$ (depending of $m$ ) with $\psi\left(F_{\lambda}, x\right) \in X-B_{\varepsilon}^{m}(y)$ for each $\left(F_{\lambda}, x\right) \geq\left(F_{\lambda_{0}}, x_{0}\right)$. But $F_{\lambda_{0}}=\left\{\psi\left(F_{\lambda_{0}}, x\right): x \in F_{\lambda_{0}}\right\}$, so $B_{\varepsilon}^{m}(y) \subset G_{\lambda_{0}}$. Let $\mathcal{P}$ the collection of all finite subsets of $X$. Then, as $\mathcal{G}$ is directed, the cover $\mathcal{V}=\left\{\bigcup_{y \in P} B_{\varepsilon}^{m}(y): P \in \mathcal{P}, m \in \mathbf{N}\right\}$ is a uniformly directed refinement of $\mathcal{G}$, since clearly $\mathcal{V}_{f}^{\varepsilon}$ refines $\mathcal{V}$. And therefore, by Theorem $31, X$ is uniformly strongly paracompact.

Conversely, suppose that there exists a cofinally Bourbaki-Cauchy net $\left(x_{\lambda}\right)_{\lambda \in \Lambda}$ in the uniformly strongly paracompact metric space $(X, d)$ that does not cluster. Then $\bigcap_{\lambda \in \Lambda} F_{\lambda}=\emptyset$, where $F_{\lambda}=\overline{\left\{x_{\nu}: \nu \geq \lambda\right\}}$, for $\lambda \in \Lambda$. Now taking $G_{\lambda}=X-F_{\lambda}$, for $\lambda \in \Lambda$, we have that $\mathcal{G}=\left\{G_{\lambda}: \lambda \in \Lambda\right\}$ is a directed open cover of $X$. Again, by Theorem 31, this cover $\mathcal{G}$ has a uniformly directed refinement $\mathcal{V}$, for which there is 
$\delta>0$ with $\mathcal{V}_{f}^{\delta}$ refining $\mathcal{V}$. On the other hand, since $\left(x_{\lambda}\right)_{\lambda \in \Lambda}$ is a cofinally BourbakiCauchy net, there exists $m \in \mathbf{N}$ and a cofinal subset $C \subset \Lambda$ such that for some $x \in X, x_{\lambda} \in B_{\delta}^{m}(x)$ for every $\lambda \in C$. Let $V \in \mathcal{V}$ such that $x \in V$. Since $\mathcal{V}_{f}^{\delta}$ refines $\mathcal{V}$, we have that $B_{\delta}^{m}(x) \subset V^{\prime}$ for some $V^{\prime} \in \mathcal{V}$ (note that $\{x\}^{\delta}=B_{\delta}(x) \subset V^{\delta}$ ). Hence $x_{\lambda} \in B_{\delta}^{m}(x) \subset G_{\lambda_{0}}$ for every $\lambda \in C$ and some $G_{\lambda_{0}} \in \mathcal{G}$ because $\mathcal{V}$ refines $\mathcal{G}$. But this contradicts the fact that $\left\{x_{\lambda}: \lambda \geq \lambda_{0}\right\} \subset F_{\lambda_{0}}=X-G_{\lambda_{0}}$. So the net $\left(x_{\lambda}\right)_{\lambda \in \Lambda}$ clusters, and therefore $X$ is cofinally Bourbaki-complete.

Note that from above result, we can say that condition (4) in Theorem 28 gives a characterization of uniformly strongly paracompactness in the setting of metric spaces by means of the set of points with no locally compact neighborhood, question that was proposed by Hohti in [22].

Next, we will give our first result about cofinal Bourbaki-complete metrization, for metrizable spaces. We will use again the set $n l c(X)$ of points with no locally compact neighborhood in order to obtain a theorem in the line of the given by Romaguera in [31] for cofinal complete metrization.

Theorem 33. For a metrizable space $X$, the following statements are equivalent:

(1) $X$ is cofinally Bourbaki-complete metrizable.

(2) The set $n l c(X)$ is compact and $X$ is strongly paracompact.

Proof. $(1) \Rightarrow(2)$ It is clear that if $X$ is cofinally Bourbaki-complete metrizable, then it is cofinally complete metrizable and then $n l c(X)$ is compact (Romaguera [31]). Moreover, from Theorem 32, $X$ is uniformly strongly paracompact, and then strongly paracompact.

$(2) \Rightarrow(1)$ Suppose that $n l c(X)$ is empty. Then $X$ is locally compact and, for every $x \in X$, let $V^{x}$ be an open neighborhood of $x$ with compact closure. Applying the Dugundji's metrization theorem, there exists a compatible metric $d$ on $X$ such that, for every $x$, the open ball $B_{1}(x)$ is contained in some compact set. Then $(X, d)$ is uniformly locally compact, and hence cofinally Bourbaki-complete (Theorem 13).

Otherwise, assume that $n l c(X)$ is non-empty. Since $n l c(X)$ is compact, there exists a countable family of open sets $\left\{W_{1}, \ldots, W_{k}, \ldots\right\}$ in $X$ such that, for every open subset $A$ of $X$ containing $n l c(X)$ there exists $k \in \mathbf{N}$ satisfying that $n l c(X) \subset W_{k} \subset A$. For example, consider $W_{k}=n l c(X)^{1 / k}, k \in \mathbf{N}$. Now, for every $x \notin n l c(X)$ take $V^{x}$ an open neighborhood of $x \in X$ with compact closure. For every $k \in \mathbf{N}$, let $\Omega_{k}$ be the open cover of $X$ given by $\Omega_{k}=\left\{V^{x}: x \notin W_{k}\right\} \cup\left\{W_{k}\right\}$. By strong paracompactness of $X$, let $\Upsilon_{k}$ be a star-finite refinement of $\Omega_{k}, k \in \mathbf{N}$. Using again the result by Dugundji, there exists a metric $d$ compatible with the topology of $X$ such that the cover of the open balls $\left\{B_{1 / k}(x): x \in X\right\}$ refines $\Upsilon_{k}$, for every $k \in \mathbf{N}$. Now, let $x \in X$ and $k \in \mathbf{N}$, we are going to see that, for every $m \in \mathbf{N}$, we can choose a finite number of members in $\Omega_{k}$, whose union contains $B_{1 / k}^{m}(x)$. Indeed, this is clear for $m=1$ since the cover $\left\{B_{1 / k}(x): x \in X\right\}$ also refines $\Omega_{k}$. Now, for $m=2$, we have that

$$
B_{1 / k}^{2}(x)=\bigcup\left\{B_{1 / k}(y): y \in B_{1 / k}(x)\right\} \subset \bigcup\left\{G \in \Upsilon_{k}: G \cap B_{1 / k}(x) \neq \emptyset\right\} .
$$

Since $B_{1 / k}(x)$ is contained in some element of the star-finite cover $\Upsilon_{k}$, we follows that the number of elements $G$ in $\Upsilon_{k}$ such that $G \cap B_{1 / k}(x) \neq \emptyset$ must be finite. Then, we can select a finite number of members in $\Omega_{k}$ whose union contains $B_{1 / k}^{2}(x)$. Next, reasoning by induction, we can get the same result for $B_{1 / k}^{m}(x)$, for every $m \in \mathbf{N}$. 
Let $\left(x_{n}\right)_{n \in \mathbf{N}}$ be a cofinally Bourbaki-Cauchy sequence in $(X, d)$, that is, for every $k \in \mathbf{N}$ there exist $m_{k} \in \mathbf{N}$ and some infinite $\mathbf{N}_{k} \subset \mathbf{N}$ such that for some $p_{k} \in X$, $x_{n} \in B_{1 / k}^{m_{k}}\left(p_{k}\right)$, for every $n \in \mathbf{N}_{k}$. Then, if the sequence $\left(x_{n}\right)_{n \in \mathbf{N}}$ is cofinally contained in some $V^{x}$ with compact closure, then the sequence clusters. Otherwise, from the above, we follows that for every $k \in \mathbf{N}$, the subsequence $\left(x_{n}\right)_{n \in \mathbf{N}_{k}}$ must be eventually contained in $W_{k}$. Thus, if $\left(x_{n}\right)_{n \in \mathbf{N}}$ does not cluster, then $\bigcap_{n \in \mathbf{N}} F_{n}=\emptyset$, where $F_{n}=\overline{\left\{x_{j}: j \geq n\right\}}, n \in \mathbf{N}$. Now if, for each $n \in \mathbf{N}$, we consider $G_{n}=X-F_{n}$, then $\mathcal{G}=\left\{G_{n}: n \in \mathbf{N}\right\}$ is a open cover of $X$. Since $n l c(X)$ is compact there exists a finite collection $G_{n_{1}}, \ldots, G_{n_{r}} \in \mathcal{G}$ such that $n l c(X) \subset G_{n_{1}} \cup \cdots \cup G_{n_{r}}$. Hence, for some $k \in \mathbf{N}$ we have that $n l c(X) \subset W_{k} \subset G_{n_{1}} \cup \cdots \cup G_{n_{r}}$, and this implies that $\left(x_{n}\right)_{n \in \mathbf{N}}$ must be cofinally contained in some $G_{n_{i}}, i \in\{1, \ldots, r\}$. But this is impossible, and therefore $\left(x_{n}\right)_{n \in \mathbf{N}}$ clusters.

According to last theorem, together with the above mentioned result of Romaguera in [31], we can say that a metrizable space is cofinally Bourbaki-completely metrizable if, and only if, it is cofinally completely metrizable and strongly paracompact. In particular, we deduce that the cofinal complete metric hedgehog $J(\mathfrak{m})$ is cofinally Bourbaki-completely metrizable if, and only if, $\mathfrak{m}$ is a countable cardinal. On the other hand, none infinite dimensional Banach space is cofinally completely metrizable nor cofinally Bourbaki-completely metrizable, even in the case they are separable in spite of we know that they can be metrizable by a Bourbakicomplete metric. Similarly, the space of irrational numbers I with the usual topology is Bourbaki-completely metrizable, since it is homeomorphic to $\mathbf{N}^{\mathbf{N}}$, but it is not cofinally Bourbaki-completely metrizable, neither cofinally completely metrizable since it is nowhere locally compact.

Now we ask if we can weaken the condition of strongly paracompactness in last theorem and put strongly metrizable. That is, we wonder if every strongly metrizable space for which $n l c(X)$ is compact, is strongly paracompact or equivalently, metrizable by a cofinally Bourbaki-complete metric. Recall that, as Balogh and Gruenhage show in [3], if a space $X$ is strongly metrizable then there exists an admissible metric $d$ such that for every $\varepsilon>0$ the cover $\left\{B_{\varepsilon}(x): x \in X\right\}$ is star-finite. Then if with this metric $d$ the space $X$ is also cofinally complete, then it is easy to see that $X$ is cofinally Bourbaki-complete. But we do not know if for a strongly metrizable space satisfying that $n l c(X)$ is compact, it is possible to find a cofinally complete metric satisfying also that for every $\varepsilon>0$ the cover $\left\{B_{\varepsilon}(x): x \in X\right\}$ is star-finite. For a similar topological result see Yasui [36].

We finish this section, with a result of cofinal Bourbaki-complete metrization in the same line of Theorem 23, that is, showing how these spaces are placed into their Stone-Čech compactification. For that, recall that Buhagiar and Yoshioka have studied in [10] the class of spaces, called ultracomplete, having countable character in their Stone-Cech compactification. That is, spaces $X$ for which there exists a countable family $\left\{G_{n}: n \in \mathbf{N}\right\}$ of open subsets of $\beta X$ containing $X$, and such that if $G$ is an open subset of $\beta X$ containing $X$ then $X \subset G_{k} \subset G$, for some $k \in$ N. In particular they have proved that, for a space $X$, it is equivalent to have countable character in $\beta X$ that in some compactification $c X$, or even in all of its compactifications. On the other hand, Yoshioka pointed out in [37] that this property is, for metrizable spaces, equivalent to cofinal complete metrizability. Then using all these results together with Theorem 33, as well as some characterizations of strongly 
paracompactness contained in the book of Arkhangel'skii and Ponomarev [1], we can obtain the desired result.

Theorem 34. For a metrizable space $X$ the following conditions are equivalent:

(1) $X$ is metrizable by a cofinal Bourbaki-complete metric.

(2) There is a countable family $\left\{G_{n}: n \in \mathbf{N}\right\}$ of open paracompact subspaces of $\beta X$ containing $X$, such that if $G$ is an open subset of $\beta X$ containing $X$ then $X \subset G_{k} \subset G$, for some $k \in \mathbf{N}$.

(3) There exist some compactification $c X$ of $X$ and a countable family $\left\{G_{n}: n \in\right.$ $\mathbf{N}$ \} of open paracompact subspaces of $c X$ containing $X$, such that if $G$ is an open subset of $c X$ containing $X$ then $X \subset G_{k} \subset G$, for some $k \in \mathbf{N}$.

Proof. $\quad(1) \Rightarrow(2)$ It is clear that (1) implies that $X$ is cofinally completely metrizable and then, as we can said before, $X$ has countable character in $\beta X$ (see Yoshioka [37]). Then there is a countable family $\left\{\hat{G}_{n}: n \in \mathbf{N}\right\}$ of open sets in $\beta X$, that are a basis for those open sets in $\beta X$ that contains $X$. On the other hand, since $X$ is also strongly paracompact (Theorem 33), then the family of open and strongly paracompact subsets of $\beta X$ containing $X$ is also a basis for the open sets in $\beta X$ containing $X$ (see this characterization of strongly paracompactness in [1]). Now, taking for every $n \in \mathbf{N}$, an open and strongly paracompact (and hence paracompact) $G_{n}$ in $\beta X$ with $X \subset G_{n} \subset \hat{G}_{n}$, condition (2) follows.

$(2) \Rightarrow(3)$ is obvious.

$(3) \Rightarrow(1)$ Clearly condition (3) implies that $X$ has countable character in some compactification, and then $X$ is cofinally completely metrizable (Yoshioka [37]). Then, by Theorem 33, we only need to see that $X$ is also strongly paracompact. For that we will use (twice) the following characterization of strongly paracompactness that can be seen in [1]: "Let $c X$ be a compactification of $X$, then $X$ is strongly paracompact if, and only if, for every compact $K$ in $c X-X$ there exists a star finite cover of $X$ by open sets in $c X$ whose closures does not meet $K$ ". So, let $K$ a compact set in $c X-X$, then $G=c X-K$ is an open set in $c X$ containing $X$. By the hypothesis, there is an open and paracompact $G_{k}$, such that $X \subset G_{k} \subset G$. Note that $G_{k}$ is in particular locally compact and paracompact, and then it must be strongly paracompact (see for instance [11]). Now, since $c X$ is also a compactification of $G_{k}$ and $K \subset c X-G_{k}$, then applying again the above result to the strongly paracompact $G_{k}$, we get a star finite cover of $G_{k}$, and in particular of $X$, by open sets in $c X$ whose closures does not meet $K$, and that complete the proof.

As in the previous section, we do not know if last result is true for each compactification $c X$.

Acknowledgements. We want to thank Professors A. Hohti and H. Junnila from the University of Helsinki for their valuable help in some parts of this paper. We are also grateful to the referee for his/her interesting suggestions.

\section{References}

[1] Arkhangel'skit, A. V., and V. I. Ponomarev: Fundamentals of general topology, problems and exercises. - D. Reidel Publishing Co., Dordrecht, 1984.

[2] Atsuji, M.: Uniform continuity of continuous functions of metric spaces. - Pacific J. Math. 8, 1958, 11-16. 
[3] Balogh, Z., and G. Gruenhage: When the collection of $\varepsilon$-balls is locally finite. - Topology Appl. 124, 2002, 445-450.

[4] Beer, G.: Which connected metric spaces are compact? - Proc. Amer. Math. Soc. 83, 1981, 807-811.

[5] Beer, G.: Between compactness and completeness. - Topology Appl. 155, 2008, 503-514.

[6] BeER, G.: Between the cofinally complete spaces and the UC spaces. - Houston J. Math. 38, 2012, 999-1015.

[7] Beer, G., and S. Levi: Gap, excess and bornological convergence. - Set-Valued Anal. 16, 2008, 1347-1362.

[8] Beer, G., and M. Segura: Well-posedness, bornologies, and the structure of metric spaces. - Appl. Gen. Topol. 10, 2009, 131-157.

[9] Bourbaki, N.: Elements of mathematics, general topology, part 1. - Hermann, Paris, 1966.

[10] Buhagiar, D., and I. Yoshioka: Ultracomplete topological spaces. - Acta Math. Hungar. 92, 2001, 19-26.

[11] Comfort, W. W., and S. Negrepontis: Continuous pseudometrics. - Lect. Notes Pure Appl. Math. 14, Marcel Dekker, Inc., New York, 1975.

[12] С̆ЕсH, E.: On bicompact spaces. - Ann. of Math. 38, 1937, 823-844.

[13] Conson, H. H.: The determination of paracompactness by uniformities. - Amer. J. Math. 80, $1958,185-190$.

[14] Dugundu, J.: Topology. - Allyn and Bacon, Inc., Boston, 1966.

[15] Engelking, R.: General topology. - Heldermann Verlag, Berlin, 1989.

[16] García-Máynez, A.: $\delta$-completeness and $\delta$-normality. - Topology Proc. 6, 1981, 345-349.

[17] García-Máynez, A., and A. Tamariz-Mascarúa: Topología general. - Editorial Porrúa, S.A., México, 1988.

[18] García-Máynez, A., and R. G. Wilson: Concerning the equality of $v X, \delta X$ and $\mu X$. - Ann. New York Acad. Sci. 552, 1989, 36-43.

[19] Garrido, I., and A.S. Meroño: Uniformly metrizable bornologies. - J. Convex Anal. 20, $2013,285-299$.

[20] Garrido, I., and A. S. Meroño: Some classes of bounded sets in metric spaces. - In: Contribuciones matemáticas en homenaje a Juan Tarrés, eds. M. Castrillón et al., Universidad Complutense de Madrid, Madrid, 2012, 179-186.

[21] Hejcman, J.: Boundedness in uniform spaces and topological groups. - Czechoslovak Math. J. 9, 1959, 544-563.

[22] Нонті, A.: On uniform paracompactness. - Ann. Acad. Sci. Fenn. Ser. A I Math. Diss. 36, 1981.

[23] Howes, N. R.: On completeness. - Pacific J. Math. 38, 1971, 431-440.

[24] Howes, N. R.: Modern analysis and topology. - Springer Verlag, New York, 1995.

[25] Hueber, H.: On uniform continuity and compactness in metric spaces. - Amer. Math. Monthly 88, 1981, 204-205.

[26] Jain, T., and S. Kundu: Atsuji spaces: equivalent conditions. - Topology Proc. 30, 2006, $301-325$.

[27] Musaev, D. K.: Uniformly superparacompact, completely paracompact, and strongly paracompact uniform spaces. - J. Math. Sci. (N.Y.) 144, 2007, 4111-4122.

[28] NJÅstaD, O.: On uniform spaces where all uniformly continuous functions are bounded. Monatsh. Math. 69, 1965, 167-176. 
[29] Pears, A. R.: Dimesion theory of general spaces. - Cambridge Univ. Press, Cambridge, 1975.

[30] RICE, M. D.: A note on uniform paracompactness. - Proc. Amer. Math. Soc. 62, 1977, 359-362.

[31] Romaguera, S.: On cofinally complete metric spaces. - Questions Answers Gen. Topology 16, 1998, 165-170.

[32] Smith, J. C.: Review of "A note on uniform paracompactness" by Michael D. Rice. - Math. Rev. 55, 1978.

[33] Tonder, G.: On a problem of Nagata. - Mathematica 20, 1978, 77-79.

[34] Willard, S.: General topology. - Dover Publications Inc., Mineola, NY, 2004.

[35] Woods, R. G.: The minimun uniform compactification of a metric space. - Fund. Math. 147, 1995, 39-59.

[36] YASUI, Y.: Unions of strongly paracompact spaces II. - Proc. Japan Acad. 44, 1968, 27-31.

[37] Yoshioka, I.: On the subsets of non locally compact points of ultracomplete spaces. - Comment. Math. Univ. Carolin. 43, 2002, 707-721.

Received 4 October 2013 • Accepted 14 January 2014 